\title{
Hepatic glucose sensing is required to preserve $\beta$ cell glucose competence
}

\author{
Pascal Seyer, ${ }^{1}$ David Vallois, ${ }^{1}$ Carole Poitry-Yamate, ${ }^{2}$ Frédéric Schütz, ${ }^{3}$ \\ Salima Metref, ${ }^{1}$ David Tarussio, ${ }^{1}$ Pierre Maechler, ${ }^{4}$ Bart Staels, ${ }^{5}$ Bernard Lanz, ${ }^{2}$ \\ Rolf Grueter, ${ }^{2}$ Julie Decaris, ${ }^{6}$ Scott Turner, ${ }^{6}$ Anabela da Costa, ${ }^{1}$ \\ Frédéric Preitner, ${ }^{1}$ Kaori Minehira, ${ }^{1,7}$ Marc Foretz, ${ }^{8}$ and Bernard Thorens ${ }^{1}$
}

\begin{abstract}
${ }^{1}$ Center for Integrative Genomics, University of Lausanne, Lausanne, Switzerland. ${ }^{2}$ Center for Biomedical Imaging, EPFL, Lausanne, Switzerland. ${ }^{3}$ Swiss Institute of Bioinformatics, Lausanne, Switzerland. ${ }^{4}$ Department of Cell Physiology and Metabolism, University of Geneva, Geneva, Switzerland 5INSERM UMR1011, Université Lille Nord, France, Institut Pasteur de Lille, Lille, France. ${ }^{6}$ KineMed Inc., Emeryville, California, USA. 7Department of Physiology, University of Lausanne, Lausanne, Switzerland. IInstitut Cochin, INSERM U1016, CNRS UMR8104, Université Paris Descartes, Department of Endocrinology, Metabolism and Cancer, Paris, France.
\end{abstract}

\begin{abstract}
Liver glucose metabolism plays a central role in glucose homeostasis and may also regulate feeding and energy expenditure. Here we assessed the impact of glucose transporter 2 (Glut2) gene inactivation in adult mouse liver (LG2KO mice). Loss of Glut2 suppressed hepatic glucose uptake but not glucose output. In the fasted state, expression of carbohydrate-responsive element-binding protein (ChREBP) and its glycolytic and lipogenic target genes was abnormally elevated. Feeding, energy expenditure, and insulin sensitivity were identical in LG2KO and control mice. Glucose tolerance was initially normal after Glut2 inactivation, but LG2KO mice exhibited progressive impairment of glucose-stimulated insulin secretion even though $\beta$ cell mass and insulin content remained normal. Liver transcript profiling revealed a coordinated downregulation of cholesterol biosynthesis genes in LG2KO mice that was associated with reduced hepatic cholesterol in fasted mice and reduced bile acids (BAs) in feces, with a similar trend in plasma. We showed that chronic BAs or farnesoid X receptor (FXR) agonist treatment of primary islets increases glucose-stimulated insulin secretion, an effect not seen in islets from $\mathrm{Fxr}^{-1-}$ mice. Collectively, our data show that glucose sensing by the liver controls $\beta$ cell glucose competence and suggest BAs as a potential mechanistic link.
\end{abstract}

\section{Introduction}

Hepatic glucose metabolism is highly regulated during the fed-tofast transition by changes in plasma levels of insulin and glucagon, but also by the changes in blood glucose concentrations. In the fed state, the presence of high insulin concentrations in the portal circulation favors storage of glucose in the form of glycogen and the use of glucose through the glycolytic pathway for its conversion into fatty acids. Important regulatory events activated during the absorptive phase include the transcriptional induction of glucokinase by insulin and of L-pyruvate kinase by the carbohydrate-responsive element-binding protein (ChREBP), which translocates to the nucleus following its dephosphorylation by a glucose metabolite-activated phosphatase (1). At the same time, glucose inhibits glycogen phosphorylase through inhibition of glycogen phosphorylase phosphatase, whereas glucose-6-phosphate activates glycogen synthase (2), thus favoring glycogen biosynthesis. The combination of insulin-dependent Srebp-1c and glucose-dependent ChREBP activation then induces the expression of lipogenic genes, including $A c c$, Fas, and $\operatorname{Scd} 1(1,3)$.

In the fasted state, the decrease in glycemia reduces the intracellular levels of glucose and glucose-6-phosphate, thereby favoring glycogen degradation and reducing the activation of ChREBP and the expression of L-pyruvate kinase and lipogenic genes. Higher glucagon levels favor the gluconeogenic pathway by inducing the expression of PEPCK and G6Pase that catalyzes the hydrolysis of glucose-6-phosphate into glucose, a reaction that takes place in the lumen of the ER. The last steps of glucose output

Conflict of interest: The authors have declared that no conflict of interest exists. Citation for this article: J Clin Invest. 2013;123(4):1662-1676. doi:10.1172/JCI65538. are classically described as involving glucose diffusion out of the ER into the cytosol followed by its export from the hepatocytes by facilitated diffusion through GLUT2. This view can be challenged on theoretical grounds since, as described above, cytosolic glucose suppresses glycogen phosphorylase activity, and its conversion into G6P activates glycogen synthase, thereby increasing glycogen accumulation. Glucose metabolism also induces L-type pyruvate kinase (L-PK) expression. These responses are all characteristic of the fed state. Therefore, glucose release from the ER lumen by a pathway that would compartmentalize glucose away from the cytosol would provide a regulatory advantage. In previous studies $(4,5)$, we showed that the absence of GLUT2 suppressed glucose uptake and facilitated diffusion of glucose across the hepatocytic plasma membrane. However, in the fasted state, hepatic glucose output was normal, and we provided evidence that it was released through a membrane traffic-based mechanism. Some glucose could nevertheless still diffuse into the cytosol, but could not escape through GLUT2, leading to increased intracellular G6P levels, reduced glycogen mobilization, and increased expression of L-PK (5). Thus, in the fasted state, GLUT2 was required to allow equilibration of cytosolic glucose with the extracellular glucose concentration to avoid the hepatocytes sensing a "fed" internal milieu. These conclusions were, however, obtained by studying mice with a constitutive and systemic deletion of the Glut2 gene and activation of a compensatory, unphysiological pathway could not be excluded.

Besides regulating glucose homeostasis, hepatic glucose metabolism has also been proposed to control feeding and energy expenditure. Early studies suggested that intrahepatic ATP levels may participate in the control of feeding (6-10), possibly by utilizing the same vagus nerve pathways that link hypothalamic glucose 
A

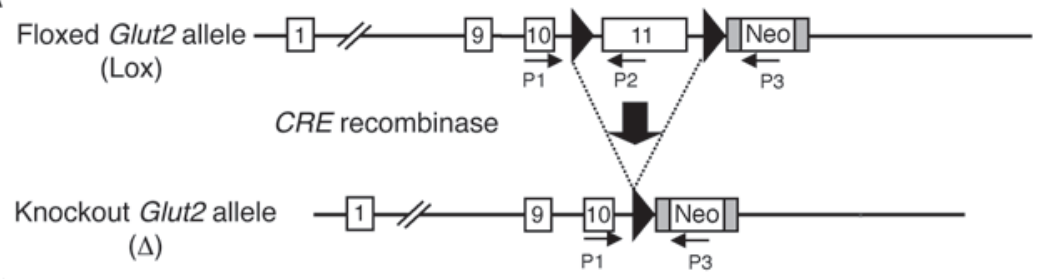

LoxP site P1, P2, P3: PCR primers

B

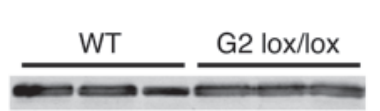

Western blot

D

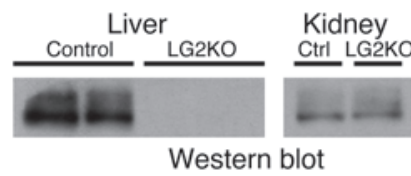

E

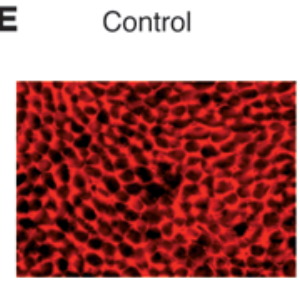

C Control
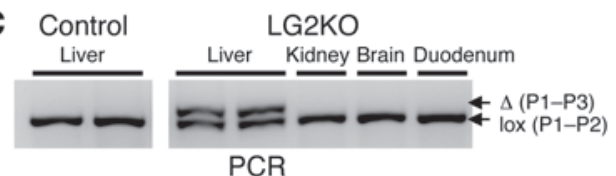

PCR

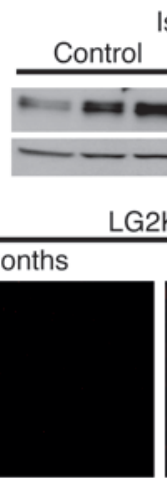

LG2KO

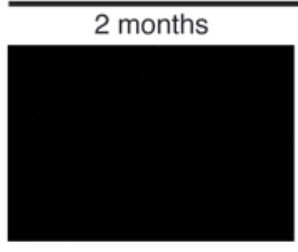

Immunofluorescence

\section{Figure 1}

Liver-specific inactivation of the Glut2 gene in adult mice. (A) Structure of the Glut2 floxed and CRE recombined alleles, and localization of the primers used for genotyping. (B) Western blot analysis of GLUT2 expression in the liver of mice with wild-type (WT) or floxed (G2 lox/lox) Glut2 alleles. (C) PCR analysis revealed the presence of the recombined Glut2 allele in the liver of tamoxifen-treated Glut2lox/lox; SA-CREERT2 (LG2KO) mice; no recombination was found in the kidney, brain, or duodenum of these same mice, nor in the liver of oil-treated Glut2/ox/lox;SA-CRE ERT2 (Control) mice. (D) Western blot analysis of GLUT2 expression in liver, kidney, and pancreatic islets of control and LG2KO mice. (E) Immunofluorescence and Western blot detection of GLUT2 in the liver of control and LG2KO mice 2 and 5 months after tamoxifen treatment. A vertical line separates nonadjacent lanes from the same blot. Scale bar: $50 \mu \mathrm{m}$. sensing to liver glucose metabolism (11). More recent work showed that overexpression of glucokinase or the bifunctional enzyme phosphofructo-2-kinase/fructose-2,6-bisphosphatase in the liver reduced feeding and body weight in obese mice. Increased activity of the bifunctional enzyme was also associated with reduced hypothalamic neuropeptide Y (NPY) expression (12).

Here, we investigated the physiology of mice with liver-specific inactivation of the Glut2 gene induced in adult mice. Our data demonstrate that this suppressed glucose uptake by hepatocytes, but had no effect on hepatic glucose output and glucose homeostasis in the fed or fasted states immediately after Glut2 gene inactivation. However, glucose intolerance developed progressively because of altered $\beta$ cell glucose competence without changes in islet insulin content and $\beta$ cell mass. Microarray analysis of liver gene expression revealed that in addition to altered regulation of glycolytic and lipogenic gene expression, all of the cholesterol biosynthesis genes had reduced expression in both fasted and fed states. This was associated with reduced hepatic cholesterol concentrations and reduced bile acid (BA) production. Because BAs increase $\beta$ cell glucose competence, we propose that their glucose-dependent production may form the basis for a liver/pancreatic islet axis to control $\beta$ cell glucose competence.

\section{Results}

Suppressed glucose uptake but normal glucose production after hepatic Glut2 inactivation. To generate mice with liver-specific deletion of the Glut2 gene, we first modified the Glut2 gene by homologous recombination in embryonic stem cells to flank exon 11 with LoxP sites. This exon encodes the last transmembrane domain and the cytoplasmic C-tail of the transporter and contains the $3^{\prime}$ untranslated region including the polyadenylation site of the Glut2 gene (Figure 1A), a targeting strategy also used for conditional inactivation of the Glut4 gene (13). The presence of the Neo cassette did not interfere with GLUT2 expression as revealed by Western blot analysis (Figure 1B). Deletion of the last transmembrane domain and C-tail of GLUT2 suppresses glucose transport activity $(14,15)$, as found for the deletion of the same transmembrane domain of GLUT8 (16) and GLUT4 (13).

To induce inactivation of the Glut2 gene in adult mouse livers, we crossed Glut $2^{l o x} /$ lox mice with $S A$-CRE ERT2 mice (17) to generate Glut $^{\text {lox } /+} ; S A-C R E^{E R T 2}$ mice, which were crossed together to obtain Glut2 $^{l o x} /$ lox $; S A-C R E^{E R T 2} / S A-C R E^{E R T 2}$ mice. Six-week-old mice were then injected with tamoxifen or oil on 3 consecutive days to induce Glut2 inactivation. As shown in Figure 1C, PCR analysis revealed the presence of the recombined Glut2 allele only in livers of tamoxifen-injected mice (LG2KO) but not in the kidney, brain, or duodenum of the same mice, nor in the liver of the oil-injected Glut2 $2^{\text {lox } / l o x} ; S A-C R E^{E R T 2} / S A-C R E^{E R T 2}$ (control) mice. The presence of the remaining floxed allele in the liver is due to the presence of non-hepatocyte cells in this organ. Two days after the end of the tamoxifen treatment, GLUT2 expression was completely eliminated in the liver of LG2KO mice, while its expression was normal in the kidney and islets (Figure 1D). Immunofluorescence and Western blot detection of GLUT2 in the liver of LG2KO mice showed no re-expression of the transporter 2 and 5 months after tamoxifen treatment (Figure 1E).

We previously reported that constitutive inactivation of the Glut2 gene led to suppressed liver glucose uptake and facilitated diffusion of glucose across the plasma membrane, but did not impair 
A

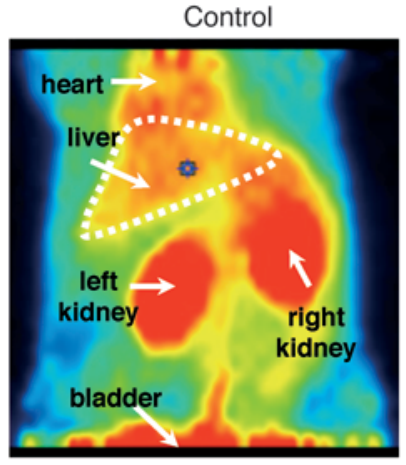

B
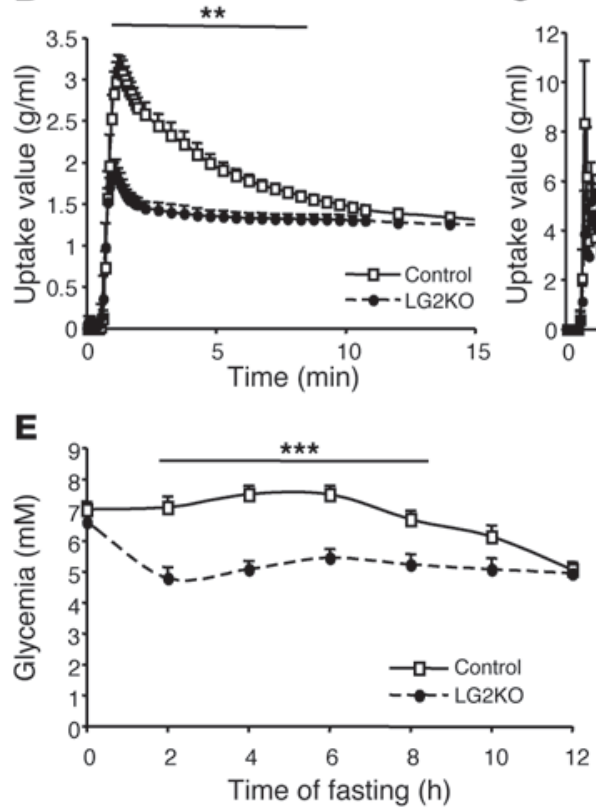

C
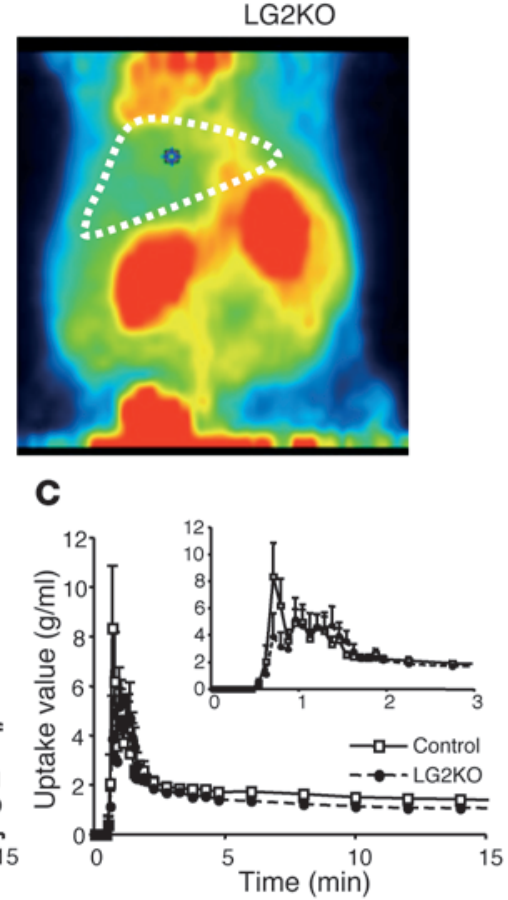

$\mathbf{F}$

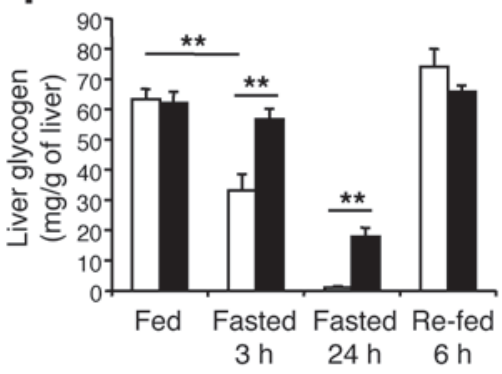

\section{Figure 2}

Absence of liver GLUT2 expression suppresses hepatic glucose uptake but does not impair endogenous glucose production. (A) PET imaging of ${ }^{18} \mathrm{FDG}$ uptake by the liver and kidneys of control and LG2KO mice during the first 15 minutes after injection (dorsal view; red, high uptake; green, low uptake). (B) Kinetics of ${ }^{18} \mathrm{FDG}$ uptake by the liver of control (white squares, $n=6$ ) and LG2KO (black circles, $n=8$ ) mice. Note the suppression of ${ }^{18} \mathrm{FDG}$ uptake between 1 minute and 9 minutes after injection in the liver of LG2KO mice. ${ }^{* \star} P<0.01$ versus control mice. (C) Time-activity curve of ${ }^{18} \mathrm{FDG}$ uptake in the blood of control (white squares, $n=6$ ) and LG2KO (black circles, $n=8$ ) mice acquired by imaging the inferior vena cava. Inset shows expanded view of the same graph from 0 to 3 minutes after tracer injection. (D) Endogenous glucose production by control and LG2KO mice $(n=10)$. (E) Evolution of blood glucose levels in fasted control and LG2KO mice $(n=9) .{ }^{* *} P<0.001$ versus control mice. (F) Evolution of hepatic glycogen content in control and LG2KO mice in the indicated states $(n=5) .{ }^{* *} P<0.01$. $\mathrm{Rd}$, rate of glucose disappearance; HGO, hepatic glucose output. hepatic glucose production (4). Here, we showed by PET scanning of ${ }^{18} \mathrm{~F}$-deoxy-D-glucose-injected ( ${ }^{18} \mathrm{FDG}$-injected) mice that glucose uptake was suppressed in the livers of LG2KO mice. This difference was not attributed to differences in the delivery of ${ }^{18} \mathrm{FDG}$ to the liver from the systemic circulation (Figure 2C). However, hepatic glucose production in 6-hour-fasted mice, as measured by glucose clamp analysis, was identical between control and LG2KO mice (Figure 2D). Nevertheless, during the early fasting period blood glucose levels decreased more rapidly in the LG2KO mice than in control mice. This was caused by an impairment in hepatic glycogen mobilization in the mutant mice (Figure 2, E and F) due to abnormal glucose and G6P intracellular levels which, respectively, reduce glycogen degradation and favor its synthesis (5).

Hepatic gene and protein expression analysis of fasted and fed mice revealed, first, that expression of the glucose-regulated transcription factor ChREBP in the nuclear fraction of LG2KO mouse livers was similar to that found in the livers of fed control mice and was maintained at this high level in the fasted state (Figure 3A). In agreement with ChREBP deregulated expression, Lpk expression was increased in the liver of fasted compared with re-fed LG2KO mice (Figure 3B). The lipogenic genes Srebp-1c, Acca, Scd1, and Elovl6, which are also in part regulated by ChREBP, also had increased expression in fasted LG2KO mouse livers compared with the control mice. However, no significant differences were noted for Fas expression. The livers of LG2KO mice were also larger than those of the control mice (Figure 3C).

Thus, these data support our previous observations using mice with systemic and constitutive Glut2 gene inactivation. They show that hepatic glucose production can proceed normally in the absence of GLUT2 and glucose uptake capacity. They show that GLUT2 is nevertheless required for normal mobilization of glycogen and for the suppression of glucose-regulated, ChREBP-dependent gene expression in the fasted state.

Normal energy homeostasis in LG2KO mice. Because there have been reports that liver glucose metabolism, in particular hepatic glycogen metabolism, may impact feeding behavior and energy expenditure (6-10), we performed extensive evaluation of energy homeostasis in control and LG2KO mice. As shown in Figure 4A, body weight gain for up to 14 weeks after tamoxifen treatment was identical between control and mutant mice. Body composition assessed by echo MRI at the end of the experiment was the same, as were body temperature, food intake, heat production, physical activity, and respiratory quotient (Figure 4, B-G). Thus, the impairment in liver glucose and glycogen metabolism induced by Glut2 gene inactivation did not have an impact on body weight, feeding, or energy expenditure. 
A

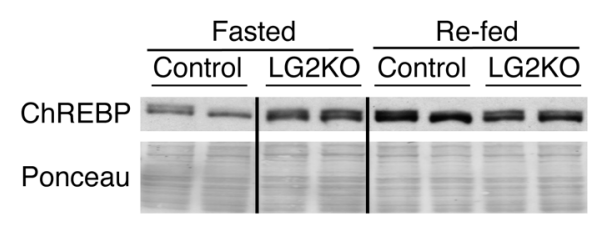

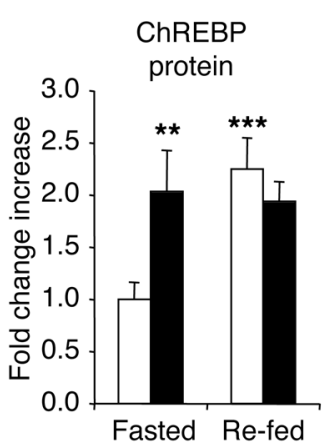
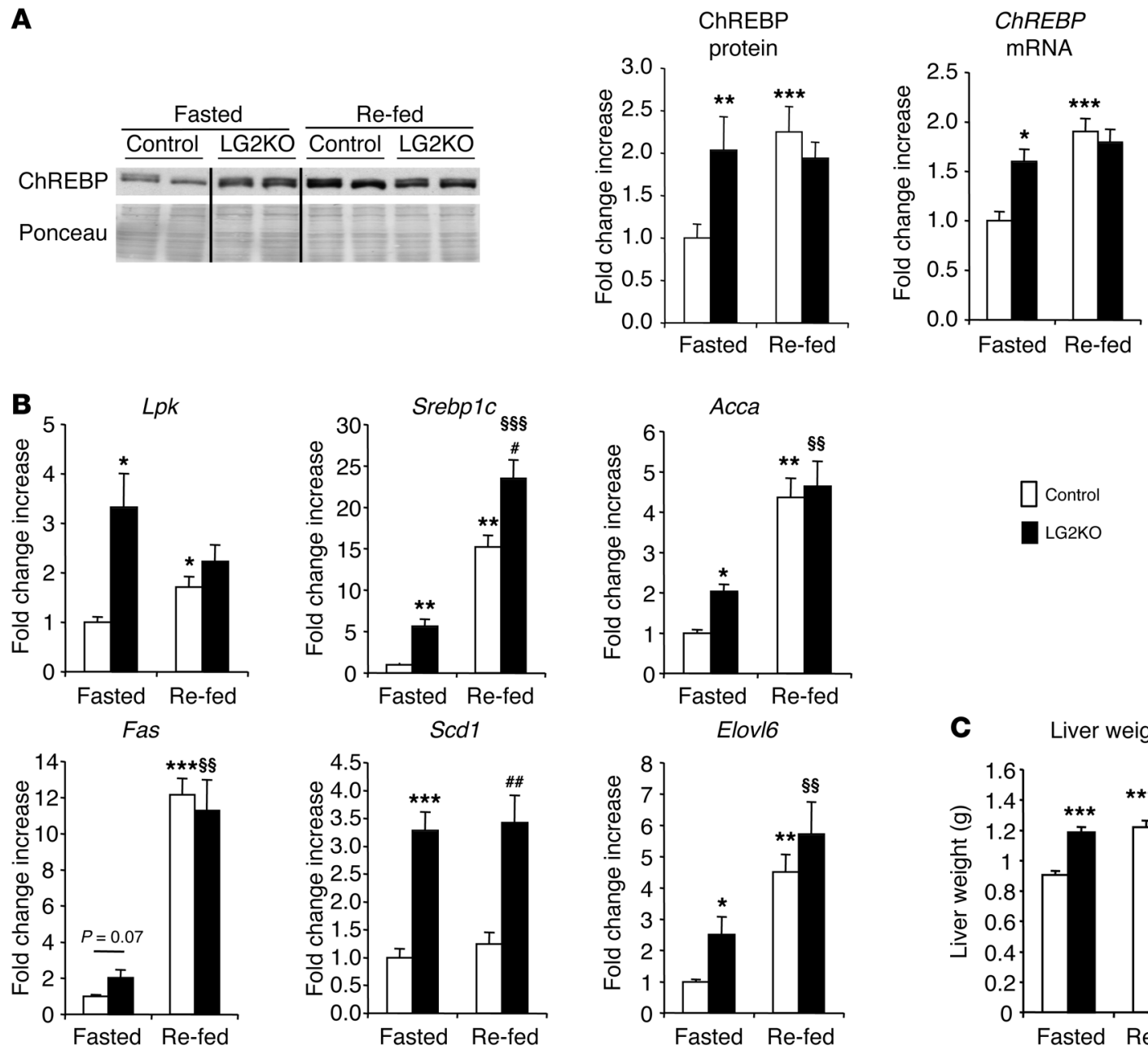

Elovl6

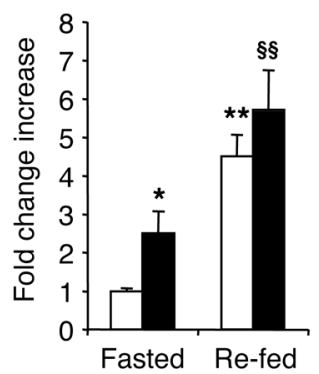

\section{Liver weight}

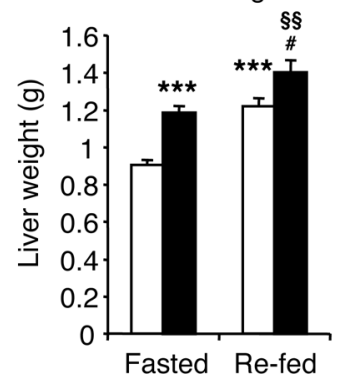

Figure 3

Abnormal regulation of glucose-sensitive genes in the livers of LG2KO mice. (A) Western blot analysis of ChREBP protein levels in nuclear fractions from the livers of 24-hour-fasted mice (Fasted) and mice re-fed for 6 hours after the end of the 24 hours of fasting (Re-fed). Vertical lines separate nonadjacent lanes from the same blot (left). Quantification of ChREBP protein levels in nuclear fraction, and ChREBP mRNA levels in the liver of fasted and re-fed control and LG2KO mice $(n=6)$ (right). (B) Levels of expression of the indicated metabolic gene mRNA in the livers of fasted and re-fed control and LG2KO mice $(n=6)$. (C) Liver weight in fasted and re-fed control and LG2KO mice $(n=12)$. Data represent the mean \pm SEM. ${ }^{*} P<0.05 ;{ }^{* \star} P<0.01$; and ${ }^{* \star *} P<0.001$ versus control fasted mice. $\$ \S P<0.01$ and $\$ \S \S P<0.001$ versus LG2KO fasted mice. ${ }^{\sharp} P<0.05$ and ${ }^{\#} P<0.01$ versus control re-fed mice.

Since liver mRNA analysis indicated increased lipogenic gene expression, we assessed hepatic triglyceride stores, de novo lipogenesis, and lipoprotein production. Triglyceride stores were lower in the fasted state in the livers of LG2KO mice compared with control mice as assessed by oil red $\mathrm{O}$ staining and biochemical measurements (Figure 5, A and B); de novo lipogenesis was decreased, although the reduction did not reach statistical significance (Figure 5C); and the rate of VLDL production was higher in the mutant mice with similar profiles of lipoprotein-associated triglycerides and cholesterol in the plasma of control and knockout mice (Figure 5, $\mathrm{D}$ and $\mathrm{E})$. Thus, there was no steatosis, despite increased lipogenic gene expression and only a moderately increased rate of VLDL export. No indication of increased beta-oxidation could be observed as measured by plasma $\beta$-hydroxybutyrate levels (not shown). Thus, the apparently surprising absence of steatosis in LG2KO mouse livers is best explained by the absence of GLUT2, which prevents the provision of glucose as a lipogenic substrate.
Progressive development of glucose intolerance in LG2KO mice. To evaluate the impact of liver Glut2 gene inactivation on glucose homeostasis, we measured glycemia and insulinemia in the fasted, fed, and fasted/re-fed state in control and LG2KO mice. This was performed 4 weeks after tamoxifen treatment, and efficient suppression of GLUT2 expression was verified by Western blot analysis for each mouse studied. As shown in Figure 6, $\mathrm{A}-\mathrm{C}$, glycemic and insulinemic levels, as well as glucose tolerance, were identical in control and LG2KO mice. Glucose-stimulated insulin secretion following i.p. glucose injection was also identical in control and LG2KO mice (Figure 6, D and E). This indicates that liver glucose uptake is not required for normal glucose homeostasis in the fed or fasted states, nor is it required for normal glucose tolerance.

To assess whether normal hepatic glucose uptake capacity is required for long-term maintenance of glucose homeostasis, we performed similar analysis 4 months after Glut2 inactivation. 
A
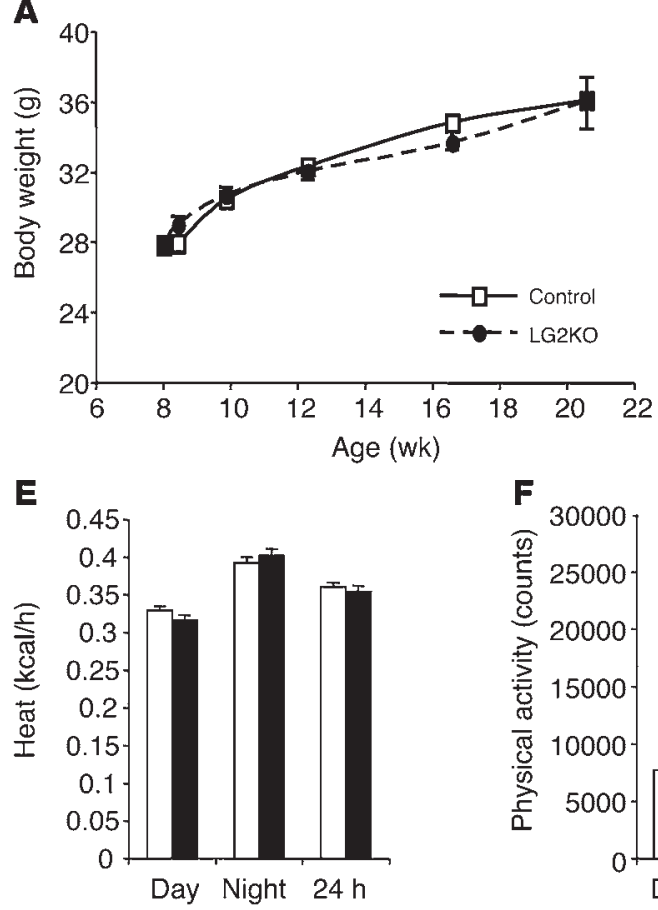

B
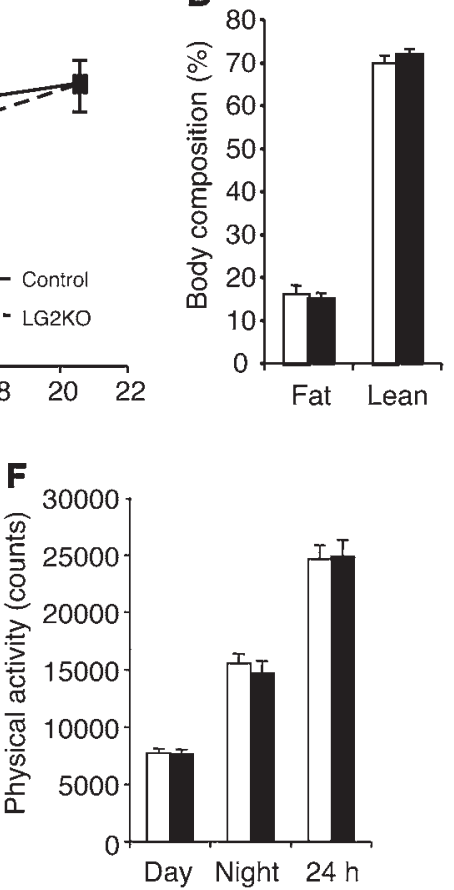
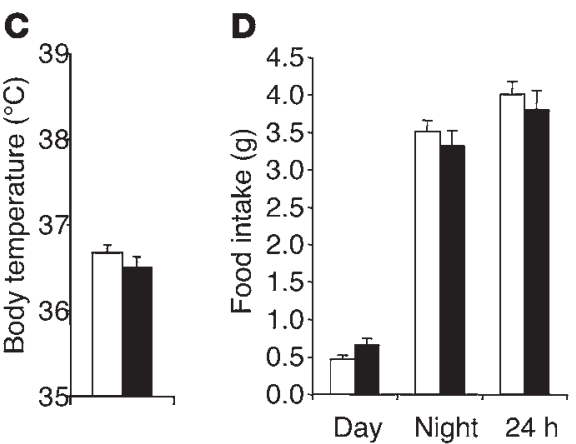

G

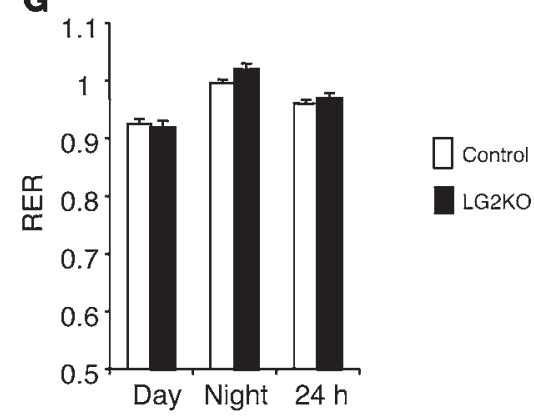

Figure 4

Normal energy homeostasis in LG2KO mice. (A) Body weight. (B) Body composition measured by echo MRI. (C) Body temperature. (D) Food intake. (E) Heat production. (F) Physical activity. (G) RER. Data represent the mean \pm SEM $(n=6-8)$.

Although there were no differences in fed, fasted, and fasted/ re-fed glycemia and insulinemia, LG2KO mice developed glucose intolerance (Figure 6, F-H). This was caused by a defect in insulin secretion, which was assessed following i.p. glucose injection (Figure 6, I and J), and was not related to a defect in plasma glucagon levels (Figure 6K), nor was it secondary to the development of insulin resistance as assessed by hyperinsulinemic-euglycemic clamp analysis in 5-hour-fasted mice (Figure 6L). However, reduced hepatic glucose output and rate of glucose disappearance were measured in basal clamp conditions, i.e., before insulin infusion (Figure 6, M and N), and were in line with the reduced mobilization of glycogen and lower glycemic levels observed early on during fasting in LG2KO mice (see Figure 2, $\mathrm{D}-\mathrm{F}$ ). In the presence of insulin, suppression of hepatic glucose output, as well as the rate of glucose disappearance, were not different between control and LG2KO mice (Figure 6, M and N). Measurements of 2-deoxy-glucose (2-DG) uptake during the hyperinsulinemic clamp revealed significantly increased uptake in the extensor digitorum longus (EDL) muscle. The uptake was higher but did not reach statistical significance in the tibialis anterior (TA) and gastrocnemius muscles, and there was no difference in uptake in the heart and perigonadal fat of control and LG2KO mice (Figure 6, O-Q). Thus, the absence of GLUT2 in the liver led to a redistribution of the infused glucose to peripheral tissues and to the development of glucose intolerance caused by reduced insulin secretion.

To further characterize the defect in insulin secretion, we performed isolated islet perifusion and pancreas perfusion experiments (Figure 7, A and B). Both experimental paradigms revealed a significant secretion defect that was present even though the insulin content per islet was identical in control and LG2KO islets, as were total pancreatic insulin content, $\beta$ cell mass, and GLUT2 expression (Figure 7, C-F). Together, these data show that the absence of GLUT2 in the liver induces progressive glucose intolerance due to a defect in $\beta$ cell glucose competence.

Hepatic glucose sensing controls a liver $/ \beta$ cell axis. Since the absence of GLUT2 from hepatocytes led to deregulated expression of glucose-sensitive glycolytic and lipogenic genes, we wondered whether other hepatic genes were also deregulated in fasted and fed conditions because of impaired glucose metabolism. We therefore performed microarray analysis of genes expressed in the liver of fasted and fed control and LG2KO mice and analyzed the expression data by gene set enrichment analysis (GSEA) (18). A single gene set that encodes the cholesterol biosynthesis enzymes behaved differently in both fasted and re-fed LG2KO mouse livers compared with control mouse livers (Figure 8A). Figure $8 \mathrm{~B}$ shows the relative gene expression of the leading edge of the gene set in both fasted and re-fed states. These data show that in both states, there was a coordinated decreased expression of most of the genes of the cholesterol biosynthetic pathway in LG2KO mice. This was confirmed for a few genes by quantitative RT-PCR analysis (qRT-PCR) (Figure 8C). Identical results were obtained 2 weeks and 4 months after tamoxifen treatment. Also, to exclude the possibility that these changes in gene expression could be the result of the tamoxifen treatment rather than Glut2 gene inactivation, we performed 2 control experiments. First, we verified that tamoxifen treatment did not affect the expression of cholesterol biosynthesis genes in Glut $2^{l o x} /$ lox mice (not shown). Second, we analyzed the expression of some of these genes in the livers of mice with constitutive Glut2 gene inactivation (Ripglut1;Glut2-/- mice, KOG2) (19). The same reduction in these gene expressions was also present (Figure 8D). 
A
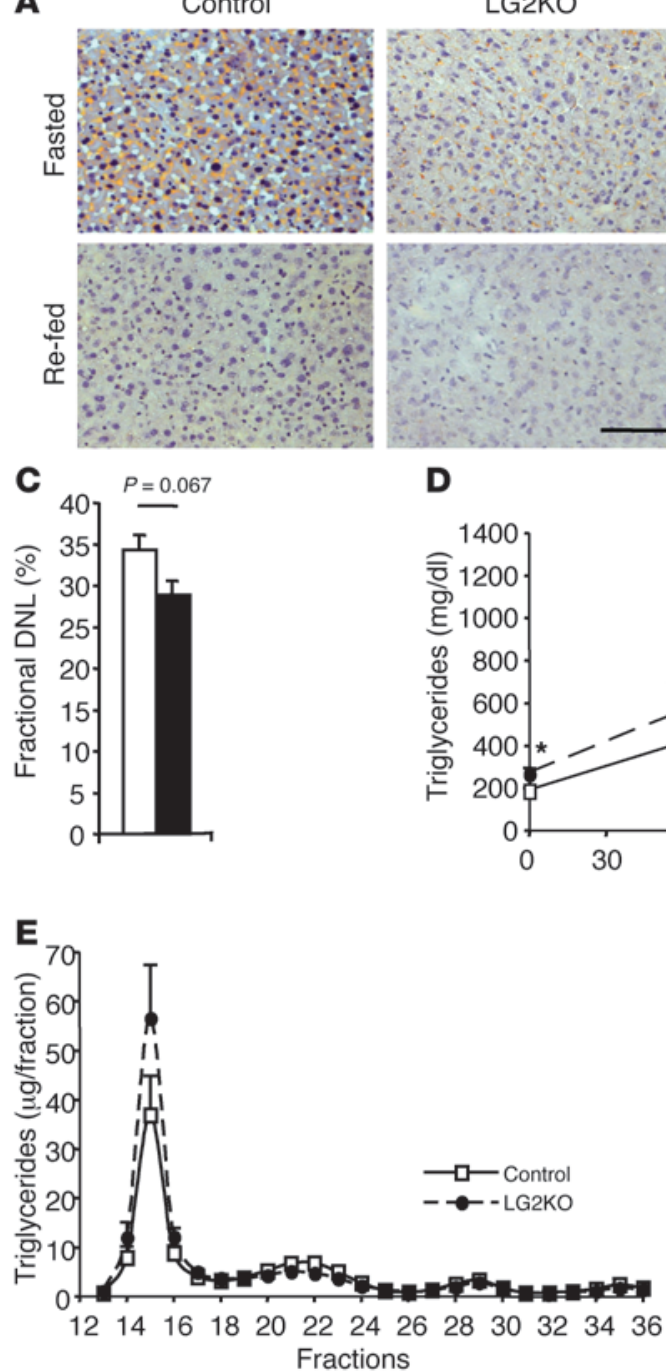

D
B
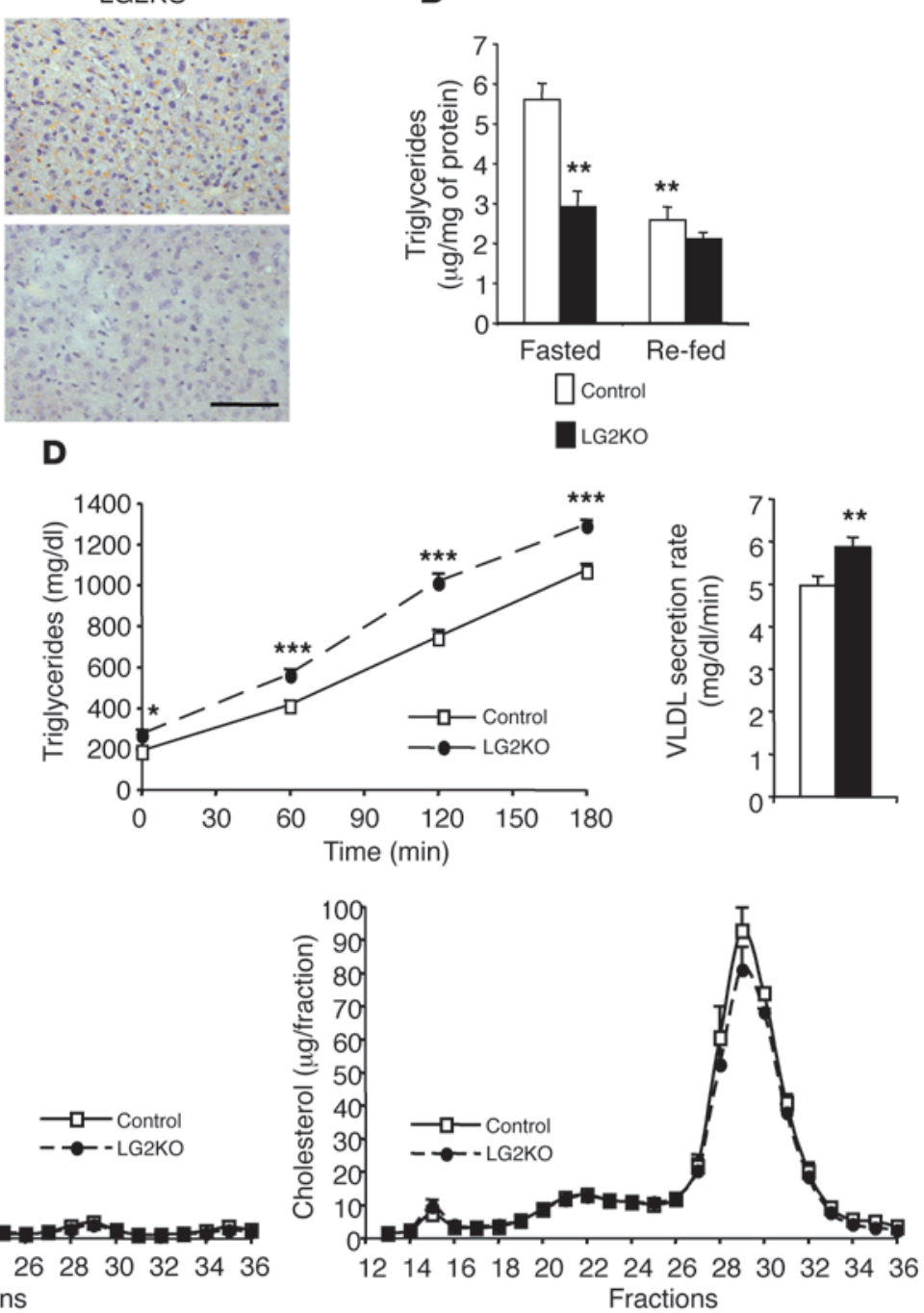

\section{Figure 5}

Impaired lipid metabolism in LG2KO mice. (A) Oil red O staining of liver sections from fasted and re-fed control or LG2KO mice. Scale bar: $50 \mu \mathrm{m}$. (B) Quantification of TG storage in the liver of control and LG2KO mice. ${ }^{* \star} P<0.01$ versus control fasted mice. (C) Fractional de novo lipogenesis measured over a 24-hour period. (D) VLDL secretion rates from overnight-fasted control and LG2KO mice. Secreted TG at the indicated time points after tyloxapol injection for control and LG2KO mice (left); rate of TG secretion derived from the left graph (right). ${ }^{*} P<0.05$; ${ }^{\star \star} P<0.01$; and ${ }^{* \star \star} P<0.001$ versus control. (E) Triglycerides (left) and cholesterol (right) contents of FPLC-fractionated plasma from 24-hour-fasted control and LG2KO mice. Data represent the mean \pm SEM $(n=6-8)$.
Biochemical measurements of cholesterol showed a 30\% decrease in cholesterol in the liver of fasted LG2KO mice compared with control mice. In the re-fed state, possibly as a result of cholesterol absorption from food, no difference in hepatic cholesterol levels was observed. In plasma, cholesterol concentrations were identical in LG2KO and control mice in either fasted or re-fed conditions (Figure 5 and Figure 9, A and B). Because cholesterol can also be converted to BAs in the liver, we measured individual and total BAs in LG2KO and control mice feces collected over 24 hours. There was a significant reduction in daily BA production in the LG2KO mice (Figure 9C) that variously affected the different individual BA species, with large reductions in deoxycholic acid, $\alpha$-murocholic acid, hyodeoxycholic acid, and $\beta$-murocholic acid (Figure 9D). In plasma, there was an approximately $30 \%$ reduction in BAs in LG2KO mice that did not, however, reach statistical significance (Figure 9E). No change was observed in the expression of genes involved in BA production (Cyp7A1, Cyp27A1, Cyp46A1, Cyp39A1, and Cyp8B1).

Previous reports have indicated that BAs may impact glucose homeostasis by a TGR5-dependent stimulation of GLP-1 secretion (20) and by direct BA action on $\beta$ cells $(21,22)$. We thus measured GLP-1 levels in the portal vein of control and LG2KO mice 30 min- utes after a re-feeding period, but could not detect any difference in GLP-1 secretion $(11.6 \pm 2.2 \mathrm{pM}$ vs. $18.4 \pm 5.2 \mathrm{pM}$; mean $\pm \mathrm{SEM}$, $n=12$ and 14 for control and LG2KO mice, respectively). We then evaluated whether BAs could acutely increase glucose-stimulated insulin secretion. We could not detect any significant effect of lithocholic acid (a preferential TGR5, rather than FXR, agonist) or of a mixture of BAs (cholic acid, taurocholic acid, deoxycholic acid, taurochenodeoxycholic acid, each at $50 \mu \mathrm{M}$ ) on $20 \mathrm{mM}$ glucosestimulated secretion, used at concentrations that are largely above their $\mathrm{K}_{\mathrm{d}}$ for TGR5 or FXR $(20,23)$ (not shown).

We next tested whether BAs could control $\beta$ cell glucose competence through treatment of islets with BAs or the GW4064 agonist of the BA receptor FXR, known to be expressed in islet cells (21). Islets were thus pre-exposed for 48 hours in the presence of CDCA (chenodeoxycholic acid) or GW4064. As shown in Figure 9F, preincubation with the BA or the synthetic agonist led to an increased stimulation of insulin secretion. This effect was dependent on FXR activity, as demonstrated by the lack of stimulation when the experiments were performed with islets from $\mathrm{Fxr}^{-/}$mice (Figure 9G). Finally, we compared the effects of pre-exposure with TGR5, FXR, and GLP-1 receptor agonists on the secretion response. Primary 

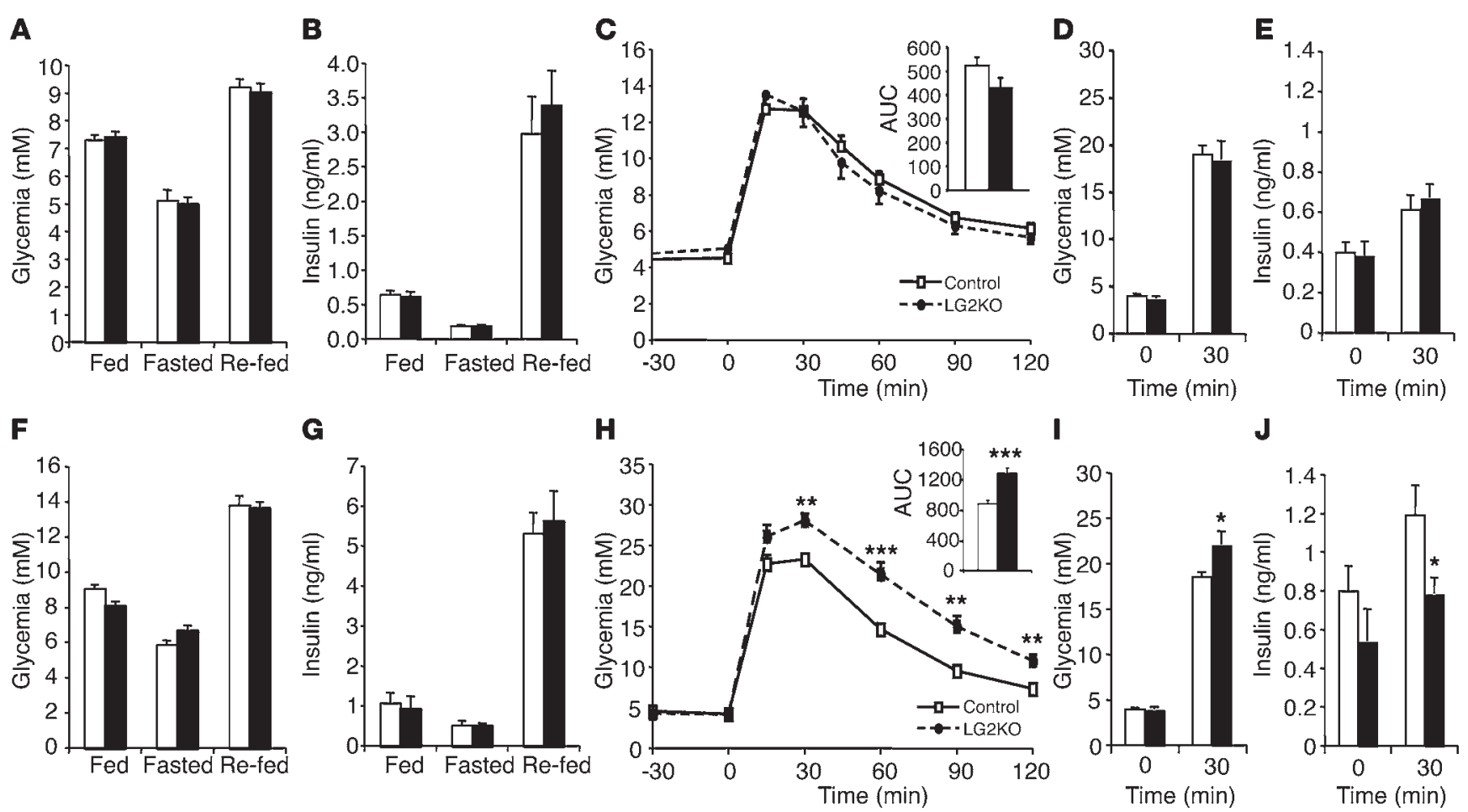

$\mathbf{J}$
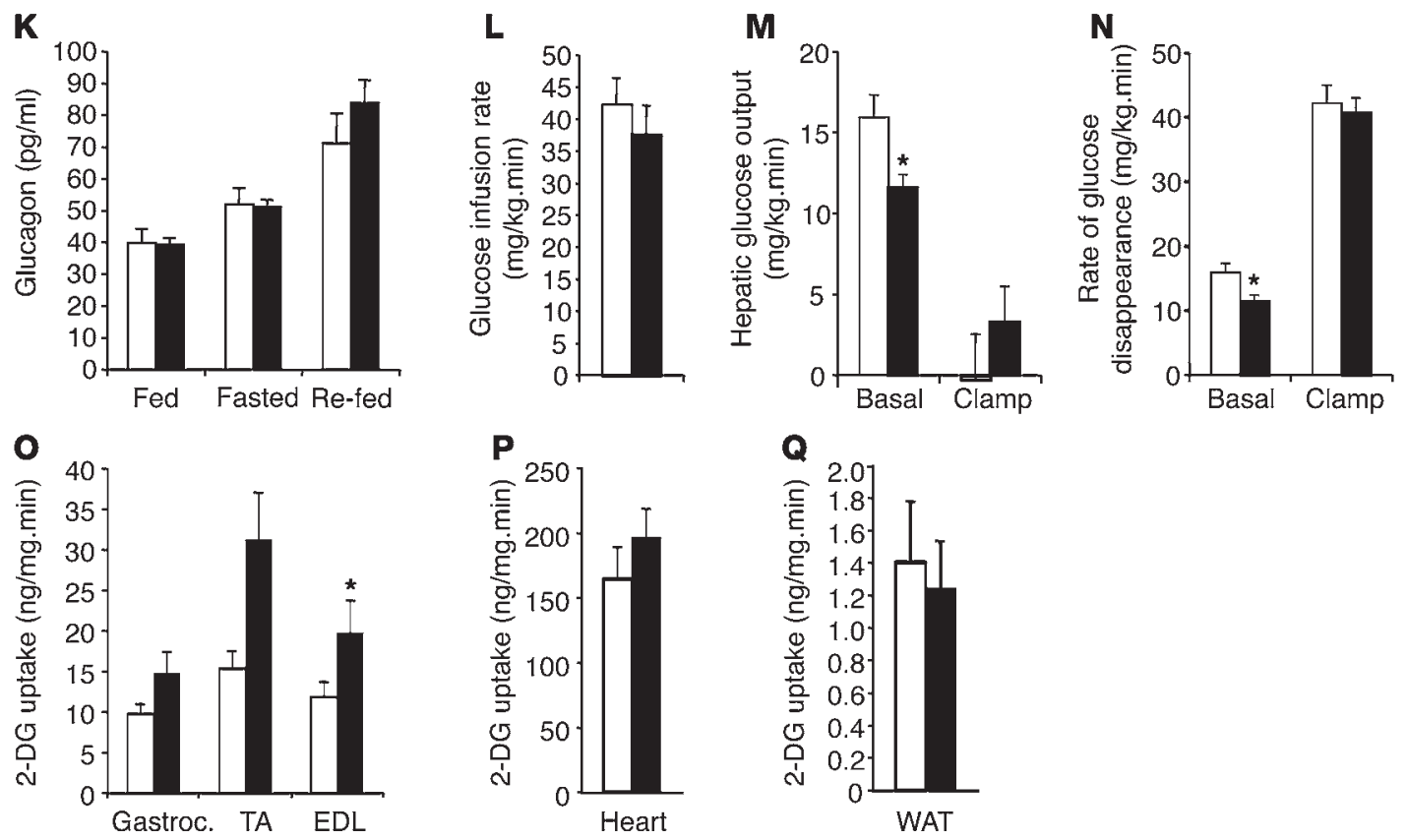

Figure 6

Progressive development of glucose intolerance in LG2KO mice. (A-C) Five days after the end of tamoxifen treatment, glycemia (A), insulinemia (B), and i.p. glucose tolerance (C) were identical in control and LG2KO mice $(n=12)$. (D) Glycemia and (E) insulinemia at the indicated time after an i.p. injection of glucose. $(\mathbf{F}-\mathbf{H})$ Four months after tamoxifen treatment, glycemia (F) and insulinemia (G) were not different in control and LG2KO mice $(n=12)$. (H) Glucose intolerance became evident following i.p. glucose injection $(n=12)$. (I) Glycemia and (J) insulinemia at the indicated time after an i.p. injection of glucose. (K) Glucagonemia $(n=6)$. For $\mathbf{A}, \mathbf{B}, \mathbf{F}, \mathbf{G}$, and $\mathbf{K}$, fasting was for 24 hours and re-feeding was for 6 hours after 24 hours of fasting. Fed mice were studied at the end of the dark period. (L) Insulin sensitivity assessed by the glucose infusion rate in hyperinsulinemic and euglycemic clamp experiments. (M) HGO before insulin infusion (Basal) and during the insulin clamp (Clamp). (N) Rate of glucose disappearance before (Basal) and during the insulin clamp (Clamp). 2-DG uptake in gastrocnemius (Gastroc.), TA, and EDL muscles $(\mathbf{O})$ in heart $(\mathbf{P})$ and in perigonadal fat (WAT) $(\mathbf{Q})$ during the insulin clamp $(n=7) .{ }^{*} P<0.05 ;{ }^{* *} P<0.01$; and ${ }^{* * *} P<0.001$ versus control. 

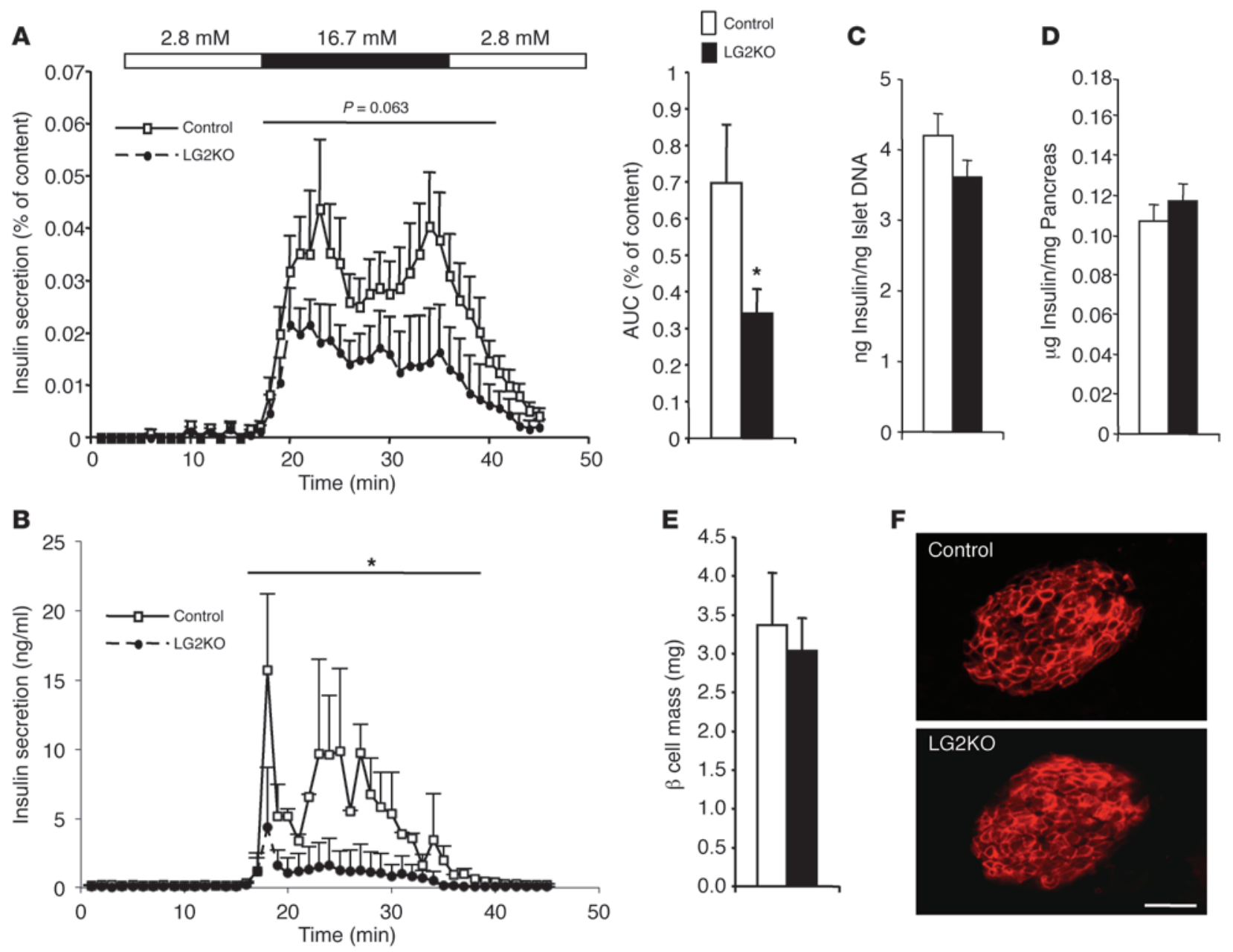

Figure 7

Reduced insulin secretory capacity in islets from LG2KO mice. (A) Four months after tamoxifen treatment, islets were isolated from control and LG2KO mice and insulin secretion was tested in perifusion experiments using the indicated glucose concentrations. AUC of the insulin secretion activity is represented on the right $(n=7)$. For AUC: Student's $t$ test, ${ }^{*} P<0.05$ versus control. (B) Pancreas perfusion experiments revealed a reduced secretory capacity of the endocrine pancreas of LG2KO mice $(n=4)$. A and B (insulin secretion graphs): 2 -way ANOVA, ${ }^{*} P<0.05$ versus control mice. (C) Insulin per islet DNA $(n=7)$. (D) Pancreatic insulin content $(n=6)$, and $(\mathrm{E}) \beta$ cell mass in control and LG2KO mice $(n=4)$. (F) Immunofluorescence detection of GLUT2 in control and LG2KO pancreatic islets. Scale bar: $30 \mu \mathrm{m}$.

islets were treated for 48 hours with RG-239 (a TGR5 agonist), CDCA, or exendin-4, and the secretion experiments were performed in the continuous presence of the indicated compounds. RG-239 did not have an impact on 20 mM GSIS, GW4064 induced approximately 4-fold increased secretion, and exendin-4 induced an approximately 21 -fold increase in secretion (Figure 9H).

The above data show that the absence of GLUT2 in the liver induced a coordinated decrease in cholesterol biosynthesis genes, which led to a decrease in BA production with no discernible impact on plasma cholesterol levels. Our results demonstrating that BAs can increase the glucose competence of $\beta$ cells through FXR-dependent mechanisms suggest that BAs could be effectors of a liver $/ \beta$ cell axis.

\section{Discussion}

Our present data show that direct glucose uptake by the liver is not critical for the control of glucose homeostasis in the fed and fasted states. However, the absence of GLUT2 impairs glycogen mobilization during fasting and prevents normal regu- lation of glycolytic and lipogenic gene expression. This is also associated with a coordinated downregulation of the cholesterol biosynthesis genes leading to lower BA production. These deregulations of liver glucose metabolism do not modify feeding behavior or energy homeostasis. However, they lead to progressive development of glucose intolerance due to a reduced insulin secretory capacity. Our data suggest that BAs may constitute a link between hepatic glucose sensing and the preservation of $\beta$ cell glucose competence.

Suppression of GLUT2 expression in hepatocytes in systemic Glut2 knockout mice was previously demonstrated to suppress glucose uptake while retaining normal hepatic glucose production. Here, using a mouse model with selective inactivation of Glut2 in the liver of adult mice, we confirm that glucose could no longer be taken up by hepatocytes, while hepatic glucose production remained unchanged in fasted mice. This confirmation of our previous data is important since it could have been argued that the suppression of Glut2 expression from birth 
A

Fasted

Enrichment plot: Cholesterol biosynthesis

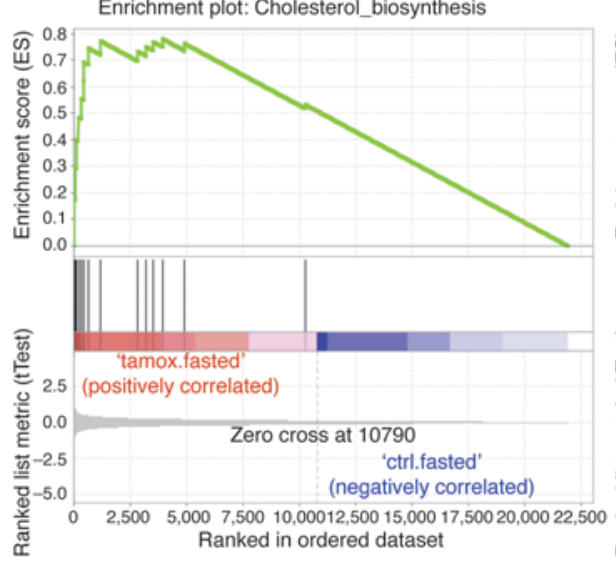

Re-fed

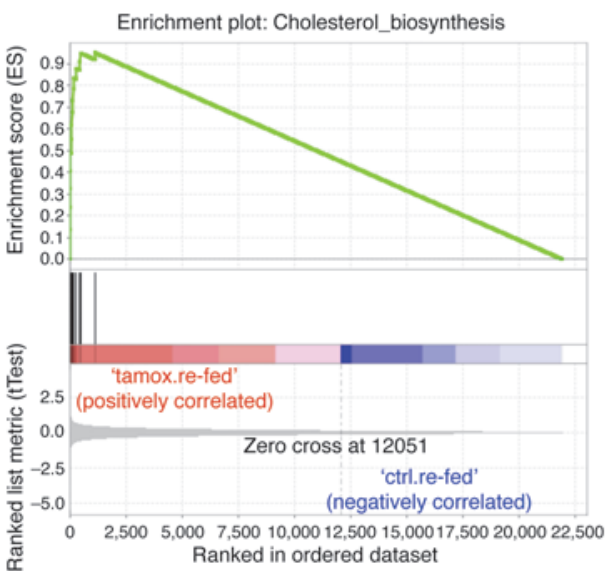

B
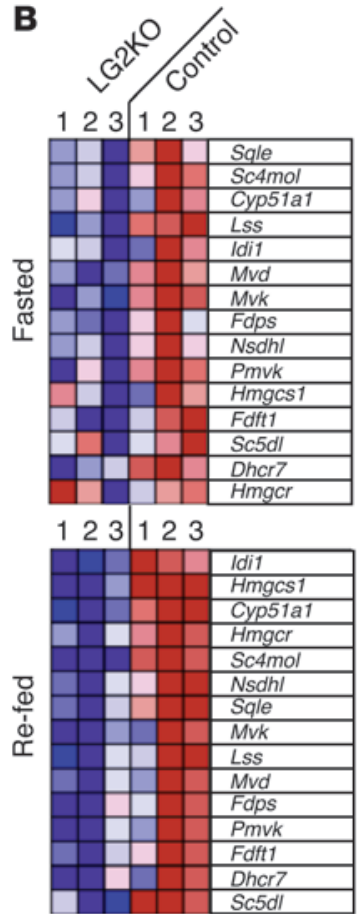

C

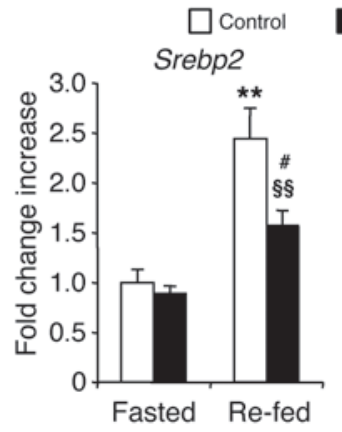

LG2KO

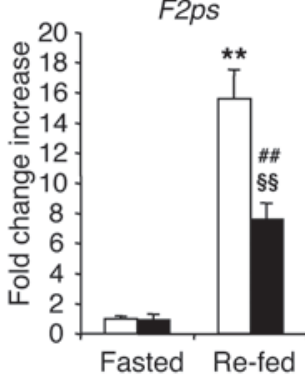

KOG2

\section{D}

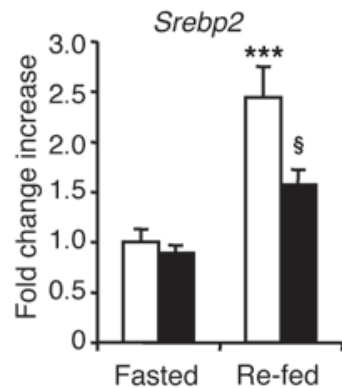

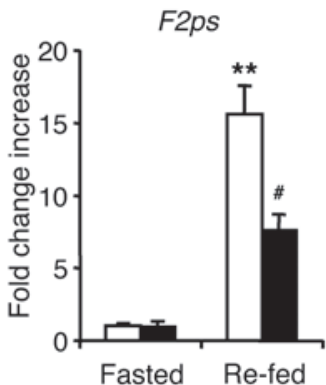
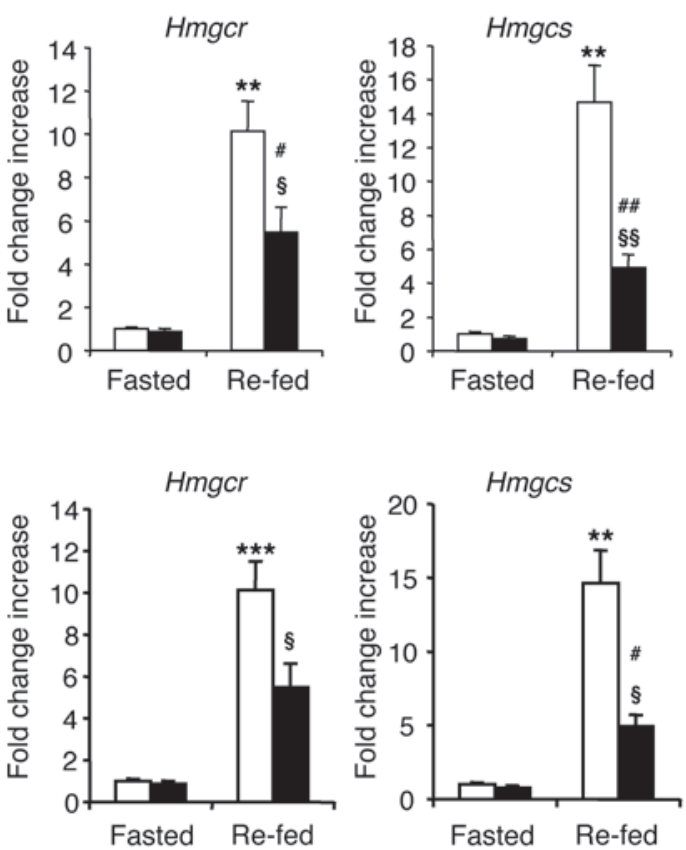

Figure 8

GSEA reveals a decreased expression of cholesterol biosynthesis genes in fasted and re-fed states. Fasting was for 24 hours and re-feeding was for 6 hours after 24 hours of fasting. (A) GSEA of microarray data from fasted and re-fed control and LG2KO mice reveal enrichment in cholesterol biosynthesis genes in both conditions. (B) Heat map representing the level of expression of genes in the leading edge of the cholesterol biosynthesis gene set (official gene symbols are used). Expression level increases from blue to red. (C) qRT-PCR analysis of the expression of the indicated cholesterol biosynthesis genes in livers of fasted and re-fed control and LG2KO mice. ${ }^{* \star} P<0.01$ versus control fasted mice. ${ }^{\S} P<0.05$

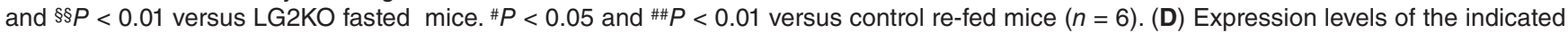
cholesterol biosynthesis genes in livers of fasted and re-fed C57BI/6 mice (B6) and mice with constitutive Glut2 knockout (Ripglut1; Glut2-/- mice backcrossed on a B6 background, KOG2). ${ }^{\star \star} P<0.01$ and ${ }^{* \star \star} P<0.001$ versus B6 fasted mice. $\$ P<0.05$ versus KOG2 fasted mice. ${ }^{\#} P<0.05$ versus $\mathrm{B} 6$ re-fed mice $(n=6)$. 
A

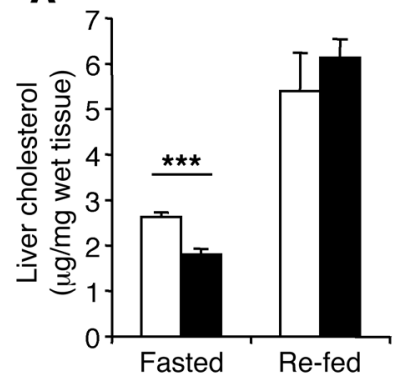

D
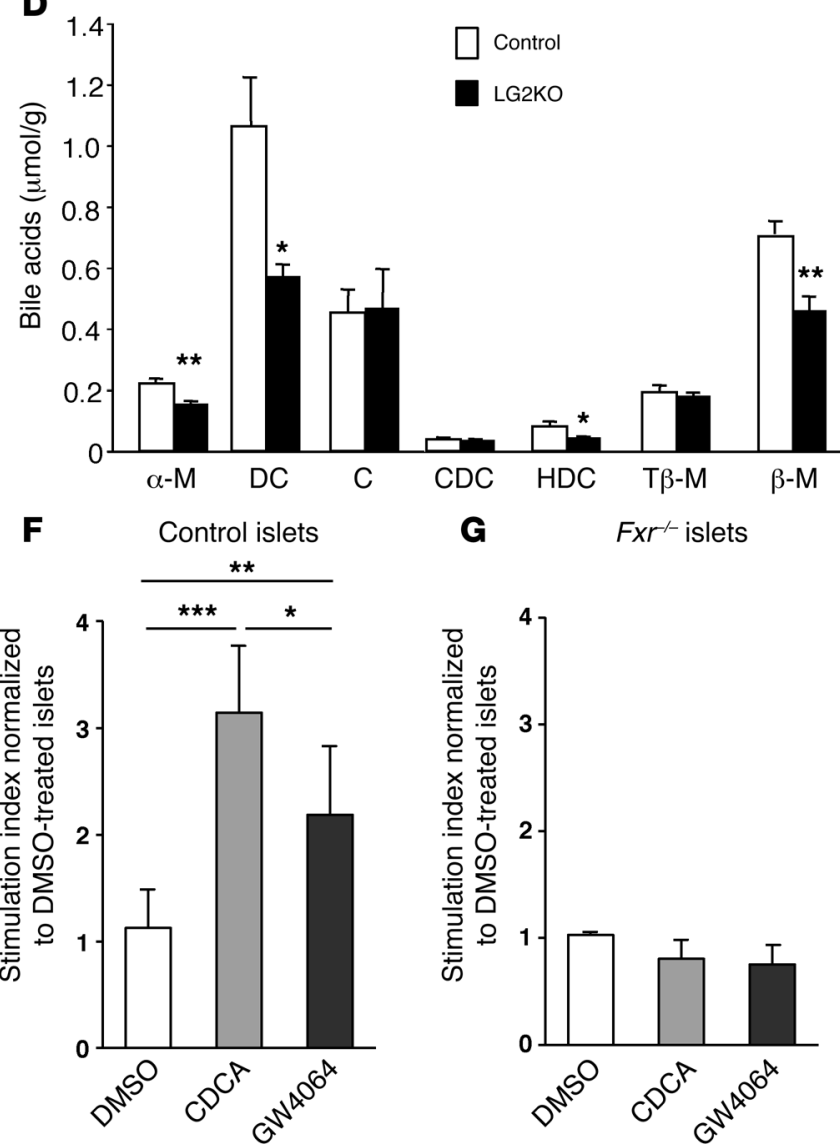

B

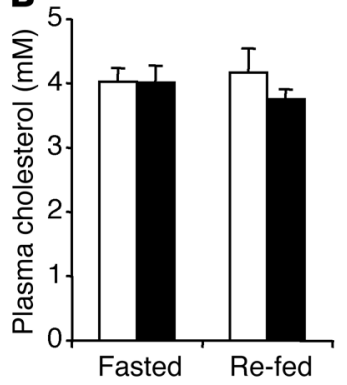

G

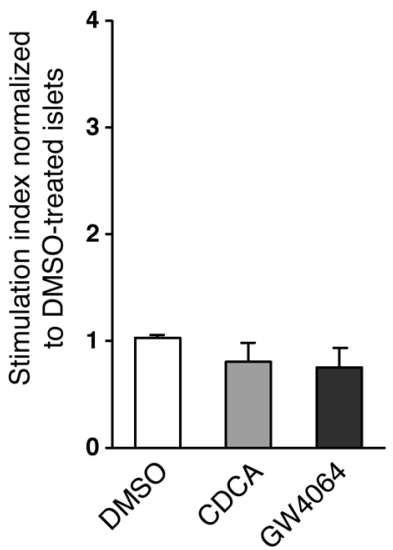

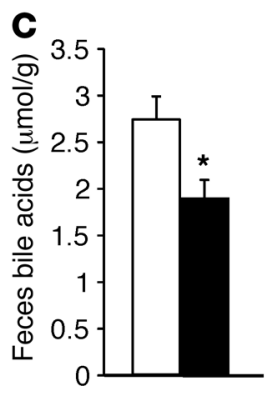

E

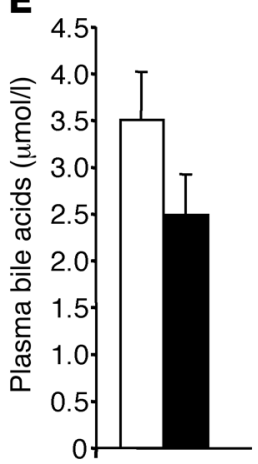

H

Control islets

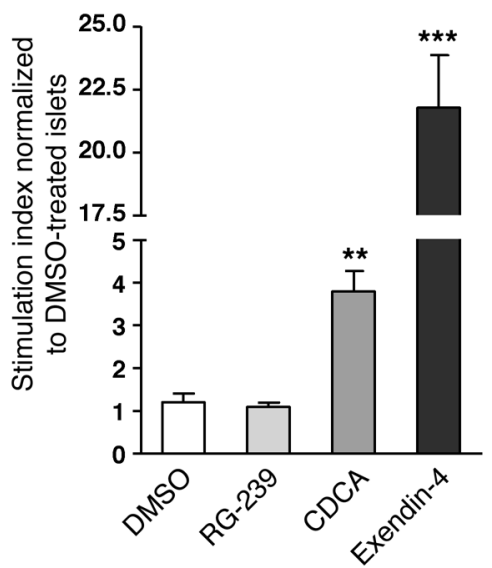

\section{Figure 9}

Reduced cholesterol and BA levels in LG2KO mice and potentiation of insulin secretion by BAs. (A) Liver cholesterol content and (B) plasma cholesterol in fasted and re-fed control and LG2KO mice $(n=6)$. (C) BA content in feces of control and LG2KO mice collected over a 24-hour period ( $n=7)$. (D) Composition of the fecal BAs: $\alpha$-murocholic acid $(\alpha-M)$; deoxycholic acid (DC); cholic acid (C); chenodeoxycholic acid (CDC); hyodeoxycholic acid (HDC); tauro- $\beta$-murocholic acid (T $\beta-\mathrm{M})$; and $\beta$-murocholic acid $(\beta-\mathrm{M})(n=7)$. (E) Total plasma BAs in the fasted state $(n=6)$. A-E: ${ }^{*} P<0.05 ;{ }^{* \star} P<0.01$; and ${ }^{* \star *} P<0.001$ versus control. (F) Twenty-four-hour pretreatment of control islets with chenodeoxycholic acid (CDCA, $50 \mu \mathrm{M})$, or the FXR agonist GW4064 $(1 \mu \mathrm{M})$ increases glucose-stimulated insulin secretion. DMSO: vehicle. (G) No potentiation of glucosestimulated insulin secretion by FXR agonists in $\mathrm{FXr}^{-1}$ islets. $(\mathrm{H})$ One-hour glucose-stimulated insulin secretion by control islets performed in the presence of, and after a 48-hour treatment with, TGR5 (RG-239), FXR (CDCA), or GLP-1 receptor (exendin-4 [Ex4]) agonists. F-H: Pool of 5 different experiments. One-way ANOVA and post-hoc Tukey's test: ${ }^{*} P<0.05$; ${ }^{* \star} P<0.01$; and ${ }^{* * *} P<0.001$. In $\mathbf{H}$, statistical significance is calculated versus DMSO-treated islets.

could lead to nonphysiological induction of an adaptive process to preserve glucose output capacity. Tamoxifen-induced Glut2 inactivation is a rapid event, taking place over a few days, and no impairment in glycemic control was observed, even in glucose tolerance tests. This indicates that hepatic glucose uptake is not a major component of glucose disposal in the absorptive phase or that redirection of glucose fluxes to skeletal muscles may rapidly compensate for impaired liver glucose uptake. The fact that fasting was associated with normal hepatic glucose production supports our previous conclusions that the release of glucose from hepatocytes may take a pathway which does not depend on facilitated diffusion through the plasma membrane, but rather 


\section{Table 1}

Primers used for quantitative RT-PCR studies

\begin{tabular}{lccc} 
Symbol & Name & Forward primer & Reverse primer \\
Acca & Acetyl CoA carboxylase isoform & TGGTGCAGAGGTACCGAAGTG & CTGCCGCAGCAGATCCAT \\
Elovl6 & ELOVL fatty acid elongase 6 & AAAGCACCCGACTAGGTGACA & ACCAGTGCAGGAAGATCAGTTC \\
F2ps & Farnesyl diphosphate synthase & ATGGAGATGGGCGAGTTCTTC & CCGACCTTTCCCGTCACA \\
Fas & Fatty acid synthase & AGGCTGGGCTCTATGGATTA & AAAAGGAGCGTCGACTTG \\
Gusb & B-glucuronidase & GTGATGGAGGAGCTGGTTCG & AGCAGAGGAAGCTCATTGG \\
Hmgcr & HMG CoA reductase & CTTGTGGAATGCCTTGTGATTG & AGCCGAGC AGC ACA TGAT \\
Hmgcs1 & HMGCoA synthetase & GCCGTGAACTGGGTCGAA & GCATATATAGCAATGTCTCCTGCAA \\
Lpk & Liver pyruvate kinase & TGGCAAGCCTGTCGTTTGT & TGGTCGAGCCTTAGTGATCA \\
Scd1 & Stearoyl CoA desaturase & CCTTCCCTTCGACTACTCTG & GCCATGCAGTCGATGAAGAA \\
Srebp1c & Sterol regulatory element binding protein 1c & GGCCGAGATGTGCGACT & TTGTTGATGAGCTGAGCATGT \\
Srebp2 & Sterol regulatory element binding protein 2 & GCGTTCTGGAGACCATGGA & ACAAATTGCTCTGAAACAAATCA \\
\hline
\end{tabular}

utilizes a distinct pathway that originates from the ER lumen where the active site of glucose-6-phosphatase is located and that depends on a membrane traffic-based mechanism.

The presence of GLUT2 in the hepatocyte membrane is nevertheless important in the fasted state, as it allows glucose equilibrations between the cytosolic compartment and the extracellular milieu. Indeed, after hydrolysis of glucose-6-phosphate in the ER, some of the glucose returns to the cytosol, and in the absence of GLUT2 it cannot escape the cells and is again converted to G6P, leading to increased G6P intracellular concentrations in the fasted state (5). This leads to reduced glycogen degradation since glucose is an inhibitor of glycogen phosphorylase and G6P is an activator of glycogen synthase. Thus, suppressed glucose equilibration between the intracellular space and extracellular milieu in the fasted state leads to permanently elevated intracellular levels of glucose and G6P, which explains the elevated expression of ChREBP in fasted LG2KO livers. This can, in turn, explain the elevated expression of glycolytic and lipogenic genes. In contrast to what might have been expected from ChREBP overexpression (24), there was no steatosis in the liver of LG2KO mice, and VLDL secretion was only moderately increased. This observation could, however, be explained by the lack of glucose uptake by hepatocytes, a major substrate for lipogenesis. Direct measurements indeed showed that de novo lipogenesis was no greater in LG2KO livers than in control mouse livers.

Impaired hepatic glucose and glycogen metabolism have been proposed to generate signals that could impact feeding control and energy homeostasis. Here, no modifications were observed in feeding, body weight and body composition, heat production, locomotor activity, or insulin sensitivity.

Together, these observations indicate that, in the absorptive phase, glucose uptake by hepatocytes is not critical for whole-body glucose and energy homeostasis. This is in apparent contrast to most textbook descriptions of the role of liver in glucose disposal in the absorptive phase. However, several studies in humans and dogs have attempted to assess splanchnic, i.e., mostly liver, glucose disposal after glucose or mixed-meal ingestion. Although quite variable, these studies proposed that approximately $10 \%-25 \%$ of the ingested glucose is taken up by the liver (25-28). In the absence of hepatic glucose absorption, the amount of glucose that would normally be taken up by the liver appears to be redirected to skeletal muscles.

Despite the lack of an acute effect of hepatic Glut2 inactivation on glucose homeostasis, long-term observations showed the progressive development of glucose intolerance. This was due to reduced insulin secretion by $\beta$ cells, whose total mass and insulin content remained normal. This therefore suggests the existence of a liver $/ \beta$ cell axis that depends on normal liver glucose metabolism and correct regulation of gene expression by glucose. That hepatic glucose metabolism may influence $\beta$ cell function was previously noted in a study of mice with liver-specific knockout of the glucokinase gene; in the basal state, mutated and control mice insulinemia was not different, but was reduced by approximately $70 \%$ in the knockout mice during hyperglycemic clamps (29). GSEA of genes expressed in control and LG2KO livers revealed that besides the deregulation of glycolytic and fatty acid synthesis genes, there was a coordinated downregulation of most of the cholesterol biosynthesis genes, both in fasted and re-fed states. Quantitative analysis showed lower expression of Srebp-2 in the re-fed, but not in the fasted, state in LG2KO mice compared with control mice, suggesting that posttranslational modifications, such as phosphorylation, acetylation, or O-glycosylation $(30,31)$, may also contribute to a change in SREBP activity. Understanding the link between hepatic glucose sensing and control of cholesterol biosynthesis genes will clearly require further investigation.

The decrease in cholesterol biosynthesis gene expression was associated with a $30 \%$ reduction in hepatic cholesterol content. This was observed in the fasted but not the re-fed state, and plasma cholesterol levels were the same between LG2KO and control mice in all conditions, suggesting that some of this cholesterol could come from absorbed food. Cholesterol is also the precursor for BA biosynthesis (32), and fecal BAs were reduced in LG2KO mice. No changes were observed in Cyp7A1 gene expression, coding for the enzyme that catalyzes the first committed step in BA production, or in the expression of other BA synthesis genes. This suggests, therefore, that a reduction in cholesterol production is the primary cause of reduced BAs in feces and plasma. Since BAs have been recently proposed to play an important role in the control of glucose homeostasis through TGR5-dependent stimulation of GLP-1 production (20), and since GLP-1 can increase $\beta$ cell glucose competence (33), we tested GLP-1 plasma levels in the basal state and after re-feeding. No differences were found between the control and LG2KO mice, suggesting that GLP-1 secretion does not explain the decreased $\beta$ cell glucose competence. In contrast, our data provide support for a direct role of BA action in $\beta$ cells through an FXR-dependent mechanism, since glucose competence of control islets was 
clearly increased by prior exposure to CDCA or the FXR agonist GW4064, an effect that was suppressed in $\mathrm{Fxr}^{-/}$islets. This is in agreement with observations that BAs increase insulin production and secretion, and also reduce islet ER stress through longterm transcriptional effects $(21,22)$. However, it is worth noting that the effect on glucose competence of 48-hour pre-exposure of primary islets to FXR agonists was much lower than that induced by GLP-1. Thus, the trophic effect of BAs on $\beta$ cell function is of relatively low potency, which is compatible with the fact that glucose intolerance became obvious only several weeks after hepatic Glut2 inactivation. Also, a recent study (34) demonstrated an acute stimulation of insulin secretion by BAs through an FXR-dependent regulation of $\mathrm{K}_{\mathrm{ATP}}$ channels. This observation is, however, different from ours since we could not find any acute effect of BAs on glucose-stimulated insulin secretion, but instead found a marked adaptation of $\beta$ cell glucose competence after prolonged incubation with BAs.

Our present data may appear to contradict some recent studies showing that BA sequestrants improve glucose homeostasis in different experimental conditions (35). For instance, treatment with colesevelam improves glucose homeostasis in diabetic mice, but this occurs without changing the total BA pool $(36,37)$. There is also evidence that improvement of glucose homeostasis upon sequestrant treatment is mediated by increased GLP-1 secretion induced by the ingested fat that is not absorbed when intestinal BAs are reduced (38). Thus, there are different mechanisms by which modulation of BA pools can impact glucose homeostasis. In addition, BAs form a family of structurally related but very diverse molecules (39) that may have widely different biological actions. Also, our present study provides an alternate mode of action of BAs on $\beta$ cell glucose competence that is observed only in long-term studies. Thus, much more work will be required to fully elucidate the role of BAs on diverse aspects of the control of glucose homeostasis. Finally, since cholesterol is the precursor of other biologically active substances, such as isoprenoid, dolichol, or steroids, it may be that other cholesterol-derived molecules also have an impact on $\beta$ cell function.

Collectively, our data show that hepatic glucose uptake depends on GLUT2 expression, but the presence of this transporter is dispensable for normal glycemic control during the absorptive phase and for a normal rate of hepatic glucose production in the fasted state. However, during fasting, GLUT2 is required for equilibration of cytosolic glucose with the external space to allow normal glycogen mobilization and suppression of glycolytic and lipogenic gene expression, processes activated by elevated glucose levels. In LG2KO hepatocytes, the permanent elevation of intracellular glucose (and G6P) leads to deregulated glucose and glycogen metabolism, but this does not affect feeding or energy homeostasis. However, in long-term studies, deregulation of liver metabolism leads to decreased $\beta$ cell glucose competence, suggesting a link between liver metabolism and $\beta$ cell function. Because the cholesterol biosynthetic pathway was the only metabolic pathway found to be downregulated, and since this was associated with decreased BA levels, we propose that BAs may mediate a liver $\beta$ cell axis by maintaining the glucose competence of these cells through an FXR-dependent mechanism. These data therefore provide evidence for an interaction between hepatic glucose handling as a means to sense glycemic levels and the long-term maintenance of an appropriate insulin secretory capacity of the endocrine pancreas.

\section{Methods}

Gene targeting and generation of liver-specific Glut2-deleted mice. The GLUT2 targeting construct was generated from genomic fragments isolated from a $129 /$ Sv mouse genomic library (40). The $5^{\prime}$ and $3^{\prime}$ homology arms, 5.4 and $3.5 \mathrm{~kb}$ in length, respectively, were inserted on either side of a PGK promoter-driven neomycin selection cassette flanked by FRT sites into the pBluescript KS plasmid. A 2-kb fragment of genomic DNA bearing exon 11, encoding the C-terminal 67 amino acids of GLUT2 including the last transmembrane domain and the intracellular C-terminal tail and flanked by loxP sites, was introduced between the $5^{\prime}$ homology arm and the neomycin-resistance cassette (Figure 1A). A PGK promoter-driven thymidine kinase (TK) cassette was added at the end of the $3^{\prime}$ homology arm. Exponentially growing HM-1 ES cells (41) were electroporated with the linearized target construct DNA and selected on plates containing G418 and ganciclovir. The targeted clones were identified by PCR across both homology arms, with confirmation by Southern blot analysis. Cell populations expanded from the targeted clones were injected into C57BL/ 6 blastocysts, and animals displaying germline transmission were mated with C57BL/6 mice. The resulting offspring were bred with $S A-C R E-E R^{T 2}$ mice, in which the coding sequence of tamoxifen-dependent CRE-ER ${ }^{\mathrm{T} 2}$ recombinase was inserted into the $3^{\prime}$ untranslated region of the serum albumin $(S A)$ gene (17), thus enabling tamoxifen-dependent CRE expression in hepatocytes. PCR analysis was used to genotype the mice. Genetic inactivation of the Glut2 gene was induced by 3 consecutive daily i.p. injections of Glut $2^{l o x} / l o x$; $S A$-CRE-ER ${ }^{T 2} / S A-C R E-E R^{T 2}$ mice with $1 \mathrm{mg}$ tamoxifen dissolved in $100 \mu \mathrm{l}$ sunflower oil (LG2KO mice). The same mice injected with oil served as controls. In some experiments, tamoxifen was injected into Glut $2^{l o x} / l o x$; $S A(W T) / S A(W T)$ mice as controls for the tamoxifen effect.

Mice, animal housing, and feeding. Animals were housed on a 12-hour light/dark cycle (0700 on, 1900 off) and were fed ad libitum a standard rodent chow (Diet 3436; Provimi Kliba AG). Mice were housed 4-5 per cage at $23^{\circ} \mathrm{C}$. For the fasting and re-feeding experiments, mice were fasted for 24 hours, or fasted for 24 hours and re-fed for 6 hours. Ripglut1; Glut $2^{-/-}$ mice (19) backcrossed for 10 generations with C57BL6 mice were used for some experiments using C57BL/6 mice as controls. $\mathrm{Fxr}^{-/}$mice have been previously described (42).

PET. In vivo measurement of hepatic glucose uptake was performed using PET. Two months after Glut2 deletion, mice were fasted for 12 or 18 hours in order to completely deplete liver glycogen stores. They were anesthetized with a mixture of $1.5 \%$ isoflurane in $100 \% \mathrm{O}_{2}(0.9 \mathrm{l} / \mathrm{ml}$, 2.5 bars) and a catheter was cannulated into the femoral or tail vein. Thereafter, mice were given a $3 \mathrm{~g} / \mathrm{kg}$ oral load of glucose $10-15$ minutes prior to PET scanning. Sixty-minute dynamic scans were acquired with the field of view centered on the mouse midsection. ${ }^{18} \mathrm{FDG}(\sim 60 \mathrm{MBq})$ was injected as a bolus through the femoral or tail vein catheter within the first $10 \mathrm{sec}-$ onds of the PET scan, followed by 300 to $500 \mu \mathrm{l}$ of saline chase solution. Imaging was performed using an avalanche photodiode microPET scanner (LabPET4; Gamma Medic). During the entire scanning period, mice were maintained under $1 \%$ isoflurane anesthesia in oxygen delivered at 0.9 liters per minute using a face mask and under temperature and breathing rate monitoring. An energy window of 250 to $650 \mathrm{keV}$ and a coincidence timing window of 22.2 nanoseconds were used. The amount of ${ }^{18} \mathrm{FDG}$ activity in the field of view (FOV), which is related to the amount of single events per second detected by the scanner, was used to evaluate and control the i.v. delivery of substrate. For image reconstruction, storage of coincidence events recorded in list mode files during the PET scan were binned according to their line of response, i.e., histograms using an iterative maximum likelihood expectation maximum-based (MLEM-based) image reconstruction algorithm (43) in a cylindrical volume of $46 \mathrm{~mm}$ in diameter and $3.7 \mathrm{~cm}$ in length. Voxel size measured $0.5 \times 0.5 \times 1.2 \mathrm{~mm}$, giving a typical 
resolution of $1.2 \mathrm{~mm}$ at the center of the FOV. For temporal histogramming the datasets were sorted into 5 -second frames for 2 minutes; 30 -second frames for 3 minutes; 2 -minute frames for 10 minutes; and 5-minute frames for 35 minutes. Subsequent to correction for the different count rates of each line of response and quantitative calibration of ${ }^{18} \mathrm{FDG}$, images of accumulated intracellular ${ }^{18} \mathrm{FDG}-6 \mathrm{P}$ at steady state were quantitatively expressed using the standardized uptake value (SUV), i.e.: (mean region of interest $[\mathrm{ROI}]$ activity $\left[\mathrm{kBq} / \mathrm{cm}^{3}\right]$ )/(injected dose $[\mathrm{kBq}] /$ body weight $[\mathrm{g}]$ ). Images were corrected for nonuniformity of the scanner response, deadtime count losses, and physical decay to the time of injection. No correction was applied for attenuation and partial-volume effects. Images were analyzed with PMOD 3.0 software (PMOD Technologies). The ROI was drawn manually by optical reading of well-delineated liver tissue and used to calculate the time-activity curve for the liver, expressed in grams per milliliter. The time-activity curve of the vena cava was used to detect the precise time of bolus injection and as a control representative of the basal radioactivity in blood vessels.

Histology and immunohistochemistry. For histology analysis, livers and other tissues were fixed in $4 \%$ paraformaldehyde for 4 hours, then left overnight in $40 \%$ sucrose and kept at $-80^{\circ} \mathrm{C}$. Twenty-micrometer cryosections were prepared for $\mathrm{H} \& \mathrm{E}$ and oil red $\mathrm{O}$ staining. Immunohistochemical detection of GLUT2 was performed as described (44) using an anti-rabbit immunoglobulin secondary antibody from the Dako EnVision Plus system.

Immunoblotting. Western blot analysis of tissue membrane preparations was performed as described (44). For quantification of nuclear proteins, nuclei were purified from fresh liver as described (45). Protein concentrations were measured using the BC Assay reagent (Interchim). ChREBP antibodies were purchased from Novus Biologicals.

$R N A$ preparations and $q R T-P C R$ analysis. Total RNA was extracted using the peqGOLD TriFast method (Peqlab), and real-time RT-PCR analysis was performed using a 7500 Fast Real-Time PCR system (Applied Biosystems). Single-strand cDNA was synthesized with random hexamers (Applied Biosystems) and Superscript II reverse transcriptase (Invitrogen). RT-PCR reactions were prepared in a final volume of $10 \mu \mathrm{l}$ containing $2 \mu \mathrm{l}$ of reverse-transcribed total RNA and $5 \mu \mathrm{l}$ of Power SYBR Green PCR master mix (Applied Biosystems) in the presence of forward (F) and reverse (R) primers at $0.3 \mu \mathrm{M}$ (primer sequences are listed in Table 1). Expression values were normalized to the expression level of $\beta$-glucuronidase (Gusb).

Liver triglyceride and cholesterol content and de novo lipogenesis. Total lipids were extracted from harvested liver by a modified Folch method (46). Triglycerides (TGs) and cholesterol were separated by use of solid-phase extraction (SPE) columns (47). Isolated TGs, cholesterol, and standards were then mixed with chloroform-Triton $\mathrm{X}(1 \%)$ solution, dried under $\mathrm{N}_{2}$ gas, and dissolved into water. TG and cholesterol content were then measured by Wako Chemical kits (Wako Chemicals GmbH). De novo lipogenesis was measured by stable isotope incorporation with analysis by gas chromatography-mass spectrometry (GC-MS). Mice were labeled with an i.p. injection $\left(20 \mu \mathrm{l} / \mathrm{g}\right.$ body weight) of pure ${ }^{2} \mathrm{H}_{2} \mathrm{O}$ (Cambridge Isotope Laboratories) 24 hours before sacrifice and then given $8 \%{ }^{2} \mathrm{H}_{2} \mathrm{O}$ as drinking water until the end of the experiment. All procedures were as described (48-50).

Liver glycogen content. Liver glycogen content was determined as described previously (51). Briefly, $100 \mathrm{mg}$ of liver tissue was dissolved in 6 volumes of $1 \mathrm{M} \mathrm{NaOH}$ for 1 hour at $55^{\circ} \mathrm{C}$ and neutralized with $1 \mathrm{~N} \mathrm{HCl}$. The extract was spun down and aliquots of the supernatant were incubated in the presence of amyloglucosidase for 2 hours at $37^{\circ} \mathrm{C}$ under vigorous shaking in a $0.2-\mathrm{M}$ sodium acetate buffer. The glucose was then assayed using a Trinder glucose oxidase method (Sigma-Aldrich).

Blood parameters and plasma hormones and lipoprotein determinations. Blood glucose levels were measured using an Ascensia Breeze2 glucometer (Bayer Healthcare). Plasma concentration of total cholesterol, HDL, LDL, TGs, and lactate were measured with a Hitachi robot (Roche) according to the manufacturer's instructions. For hepatic VLDL secretion rate determination, mice were fasted overnight and $500 \mathrm{mg} / \mathrm{kg}$ of Tyloxapol (a lipoprotein lipase inhibitor; Sigma-Aldrich) was injected i.v. Blood was collected at 0, 60, 120, and 180 minutes. Total plasma TGs were determined using a commercial kit (Wako Chemicals GmbH). The distribution of lipids within the plasma lipoprotein fractions was assessed after overnight fasting by separating the plasma samples using fast protein liquid chromatography (FPLC) with a Superose 6 10/30 column (Pharmacia Fine Chemicals) (52). TGs and cholesterol were determined in each fraction using commercial kits (Wako Chemicals $\mathrm{GmbH}$ ).

To measure plasma GLP-1 levels, 16-hour-fasted mice were re-fed for 30 minutes, then anesthetized with isoflurane, and blood was immediately collected from the portal vein into tubes containing 0.28 units $/ \mathrm{ml}$ aprotinin, $12.5 \mathrm{mM}$ EDTA, $0.1 \mu \mathrm{M}$ diprotin, and kept on ice until centrifugation at $4{ }^{\circ} \mathrm{C}$. Active GLP- 1 content was measured using an RIA kit (Linco Research, Millipore). Insulin was measured using an ultrasensitive mouse insulin ELISA (Mercodia). Glucagon was measured by RIA (Linco Research).

Study of glucose metabolism and insulin sensitivity. For glucose tolerance tests, 16-hour-fasted mice were i.p. injected with glucose $(1 \mathrm{~g} / \mathrm{kg})$ and blood glucose levels were determined from tail-tip bleedings at the indicated times relative to glucose injections. In vivo glucose-stimulated insulin secretion was determined in separate experiments on plasma samples prepared 30 minutes before and 30 minutes after glucose injection. Hyperinsulinemic euglycemic clamp studies were performed on 16-week-old mice (2-month tamoxifen treatment). An indwelling catheter for insulin and glucose infusion was placed into the left femoral vein under isoflurane anesthesia. Mice were allowed to recover for 6 to 8 days until they had regained 95\%-100\% of their initial body weight. After a 5-hour fast, a 180-minute hyperinsulinemic euglycemic clamp was conducted in awake, freely moving mice, as previously described (48). HPLC-purified $\left[3-{ }^{3} \mathrm{H}\right]$-glucose (NEN Life Sciences, PerkinElmer) was prime infused throughout the clamp ( $5 \mu \mathrm{Ci}$ bolus, followed by $0.05 \mu \mathrm{Ci} / \mathrm{min}$ [basal] and $0.1 \mu \mathrm{Ci} / \mathrm{min}$ [clamp]) to estimate glucose turnover and hepatic glucose production. After an 80-minute basal period, a blood sample was collected from the tail tip for determination of basal glucose turnover. The clamp was initiated by prime infusion of human insu$\operatorname{lin}(2.5 \mathrm{mU} / \mathrm{kg} / \mathrm{min}$; Actrapid; Novo Nordisk), and $50 \%$ glucose was infused at variable rates and adjusted every 10 minutes to clamp plasma glucose levels around $5.5 \mathrm{mM}(100 \mathrm{mg} / \mathrm{dl})$ as measured in 2- $\mu$ l blood samples. With this dose of insulin, hepatic glucose production is partially suppressed (53). After a 2-hour stabilization period, a bolus $(10 \mu \mathrm{Ci})$ of 2-deoxy-D-[1-14 C]glucose $\left(2-\left[{ }^{14} \mathrm{C}\right] \mathrm{DG}\right.$; NEN Life Sciences, PerkinElmer) was injected for the determination of insulin-stimulated glucose uptake in individual tissues. Blood was sampled from the tail tip at 5, 15, 25, 35, and 45 minutes after bolus injection. At the end of the clamp study, mice were euthanized and tissues were collected and frozen in liquid nitrogen for subsequent analysis. Glucose concentrations in plasma samples were measured using a glucose oxidation method (Thermo Electron), and $\left[{ }^{3} \mathrm{H}\right]$ glucose was quantified as previously described (54). Rates of basal and clamp glucose turnover were determined as the ratio of the $\left[3-{ }^{3} \mathrm{H}\right]$ glucose infusion rate to the specific activity of plasma glucose at the end of the basal period and during the final 40 minutes of the clamp period, respectively. Hepatic glucose production was determined by subtracting the rate of glucose infusion from the rate of whole-body glucose uptake. For the determination of tissue 2-[ $\left.{ }^{14} \mathrm{C}\right] \mathrm{DG}-6-$ phosphate (2-DG-6-P) content, tissue samples were homogenized and the supernatants were passed through ion-exchange columns to separate 2-DG-6-P from 2-DG, as previously described (55).

Pancreas perfusion and islet insulin secretion. Pancreas perfusion was performed as described (56). For islet perifusion experiments, islets were isolated by hand picking after collagenase digestion of the pancreas and samesize islets were chosen for secretion experiments performed as described 
(57). At the end of each perifusion, islets were collected and lysed in acid ethanol $(75 \% \mathrm{EtOH} ; 0.55 \% \mathrm{HCl})$ for assessment of insulin content. Separate batches of islets were used to measure insulin/DNA content. For static incubations, isolated islets were transferred in 12-well plates and preincubated or not with BAs (chenodeoxycholic acid, CDCA, $50 \mu \mathrm{M}$ ) or the FXR agonist GW4064 $(1 \mu \mathrm{M})$ for 48 hours. Islets were then preincubated for 1 hour in $1 \mathrm{ml} \mathrm{KRBH}$ supplemented with $2.8 \mathrm{mM}$ glucose, then transferred to $1 \mathrm{ml} \mathrm{KRBH}$ supplemented with 2.8 or $20 \mathrm{mM}$ glucose. After 2 hours, islets and media were collected for insulin measurements.

Pancreas histology and insulin endocrine hormone determination. Mice were killed and the pancreas were quickly dissected, weighed, frozen in liquid nitrogen, and homogenized in acid ethanol. For histological analysis, whole pancreases were fixed with $4 \%$ paraformaldehyde and embedded in paraffin. Five to six sections of the whole pancreas were stained for insulin and $\beta$ cell area was measured in all sections using ImageJ software (NIH); $\beta$ cell mass was calculated by multiplying pancreas weight by the percentage of the pancreas surface occupied by $\beta$ cells.

Microarray analysis. Fasted and re-fed control and LG2KO mice were killed 15 days after tamoxifen treatment and liver RNAs were extracted using Trifast reagent (Peqlab). For each group, 3 pools of RNA from 3 animals were prepared and, following quality control, were used for hybridization to Affymetrix Mouse Genome 4302.0 arrays according to the supplier's instructions. The data were analyzed using R statistical software (58). Following normalization using the RMA package (59), $2 \times 2$ factorial analysis conducted with the Limma package (60) was used to identify differentially expressed genes, in particular those differentially regulated between control and LG2KO mice. GSEA was performed as previously described (18). Microarray data are available in the NCBI's Gene Expression Omnibus, (accession number GSE43581).
Body composition, energy homeostasis. Fat and lean body masses were determined on isoflurane-anesthesized animals using an EchoMRI whole-body composition analyzer. Indirect calorimetry was performed 1 month after Glut2 deletion using an 8-chamber Oxymax system (Columbus Instruments). Gas concentrations were monitored at the inlet and outlet of the sealed chambers. Oxygen consumption $\left(\mathrm{VO}_{2}\right)$ and carbon dioxide production $\left(\mathrm{VCO}_{2}\right)$ were calculated and used to assess energy expenditure (heat production) and respiratory quotient (RER). Mice were allowed to acclimate to the cages for 2 days before 1 or 2 cycles of 24-hour measurements. Statistics. All data are mean \pm SEM and were compared by an unpaired, 2 -tailed Student's $t$ test unless stated otherwise. A $P$ value less than 0.05 was considered significant. $P$ values are indicated in the figure legends.

Study approval. All experimental protocols were approved by the Service Vétérinaire du Canton de Vaud (Switzerland).

\section{Acknowledgments}

We thank Bert Groen and Folkert Kuipers (Groningen, The Netherlands) for BA measurements. This work was supported by a Swiss National Science Foundation grant (3100A0-113525), and the NCCR Frontiers in Genetics and the European Union 7th FP grants Hepadip and BetaBat (to B. Thorens).

Received for publication June 28, 2012, and accepted in revised form January 24, 2013.

Address correspondence to: Bernard Thorens, Center for Integrative Genomics, Genopode Building, University of Lausanne, CH-1015 Lausanne, Switzerland. Phone: 41.21.692.3981; Fax: 41.21.692.3985; E-mail: Bernard.Thorens@unil.ch.
1. Postic C, Girard J. Contribution of de novo fatty acid synthesis to hepatic steatosis and insulin resistance: lessons from genetically engineered mice. J Clin Invest. 2008;118(3):829-838.

2. Carabaza A, Ciudad CJ, Baque S, Guinovart JJ. Glucose has to be phosphorylated to activate glycogen synthase, but not to inactivate glycogen phosphorylase in hepatocytes. FEBS Lett. 1992;296(2):211-214.

3. Foufelle F, Ferre P. New perspectives in the regulation of hepatic glycolytic and lipogenic genes by insulin and glucose: a role for the transcription factor sterol regulatory element binding protein-1c. Biochem J. 2002;366(pt 2):377-391.

4. Guillam MT, Burcelin R, Thorens B. Normal hepatic glucose production in the absence of GLUT2 reveals an alternative pathway for glucose release from hepatocytes. Proc Natl Acad Sci U S A. 1998; 95(21):12317-12321.

5. Burcelin R, del Carmen Muñoz M, Guillam MT, Thorens B. Liver hyperplasia and paradoxical regulation of glycogen metabolism and glucose-sensitive gene expression in GLUT2-null hepatocytes. Further evidence for the existence of a membrane-based glucose release pathway. J Biol Chem. 2000;275(15):10930-10936.

6. Langhans W, Grossmann F, Geary N. Intrameal hepatic-portal infusion of glucose reduces spontaneous meal size in rats. Physiol Behav. 2001; 73(4):499-507.

7. Scharrer E, Rossi R, Sutter DA, Seebacher MC, Boutellier S, Lutz TA. Hyperpolarization of hepatocytes by 2,5-AM: implications for hepatic control of food intake. Am J Physiol. 1997; 272(3 pt 2):R874-R878.

8. Friedman MI. Control of energy intake by energy metabolism. Am J Clin Nutr. 1995; 62(5 suppl):1096S-1100S.

9. Stubbs RJ. Peripheral signals affecting food intake. Nutrition. 1999;15(7-8):614-625.
10. Nicolaidis S. Metabolic and humoral mechanisms of feeding and genesis of the ATP/ADP/AMP concept. Physiol Behav. 2011;104(1):8-14.

11. Pocai A, et al. Hypothalamic K(ATP) channels control hepatic glucose production. Nature. 2005; 434(7036):1026-1031.

12. Wu C, et al. Enhancing hepatic glycolysis reduces obesity: differential effects on lipogenesis depend on site of glycolytic modulation. Cell Metab. 2005; 2(2):131-140

13. Abel ED, et al. Cardiac hypertrophy with preserved contractile function after selective deletion of GLUT4 from the heart. J Clin Invest. 1999; 104(12):1703-1714.

14 . Santer R, et al. The mutation spectrum of the facilitative glucose transporter gene SLC2A2 (GLUT2) in patients with Fanconi-Bickel syndrome. Hum Genet. 2002;110(1):21-29.

15. Foretz M, Thorens B. The facilitative glucose transporter 2: pathophysiological role in mouse and human. In: Broër S, Wagner CA, eds. Membrane Transporter Diseases. New York, New York, USA: Kluwer Academic/Plenum Publishers; 2003:175-190.

16. Membrez M, et al. GLUT8 is dispensable for embryonic development but influences hippocampal neurogenesis and heart function. Mol Cell Biol. 2006; 26(11):4268-4276.

17. Schuler M, Dierich A, Chambon P, Metzger D. Efficient temporally controlled targeted somatic mutagenesis in hepatocytes of the mouse. Genesis. 2004;39(3):167-172.

18. Subramanian A, et al. Gene set enrichment analysis: a knowledge-based approach for interpreting genome-wide expression profiles. Proc Natl Acad Sci US A. 2005;102(43):15545-15550.

19. Thorens B, Guillam MT, Beermann F, Burcelin R, Jaquet $M$. Transgenic reexpression of GLUT1 or GLUT2 in pancreatic beta cells rescues GLUT2null mice from early death and restores normal glucose-stimulated insulin secretion. J Biol Chem. 2000;275(31):23751-23758.

20. Thomas C, et al. TGR5-mediated bile acid sensing controls glucose homeostasis. Cell Metab. 2009; 10(3):167-177.

21. Popescu IR, et al. The nuclear receptor FXR is expressed in pancreatic $\beta$-cells and protects human islets from lipotoxicity. FEBS Lett. 2010; 584(13):2845-2851.

22. Renga B, Mencarelli A, Vavassori P, Brancaleone V, Fiorucci $S$. The bile acid sensor FXR regulates insulin transcription and secretion. Biochim Biophys Acta. 2010;1802(3):363-372.

23. Kawamata Y, et al. A G protein-coupled receptor responsive to bile acids. J Biol Chem. 2003; 278(11):9435-9440.

24. Benhamed F, et al. The lipogenic transcription factor ChREBP dissociates hepatic steatosis from insulin resistance in mice and humans.J Clin Invest. 2012;122(6):2176-2194.

25. Capaldo B, et al. Splanchnic and leg substrate exchange after ingestion of a natural mixed meal in humans. Diabetes. 1999;48(5):958-966.

26. Ferrannini E, et al. The disposal of an oral glucose load in healthy subjects. A quantitative study. Diabetes. 1985;34(6):580-588.

27. Woerle HJ, et al. Pathways for glucose disposal after meal ingestion in humans. Am J Physiol Endocrinol Metab. 2003;284(4):E716-E725.

28. Moore MC, et al. Disposition of a mixed meal by the conscious dog. Am J Physiol. 1994; 266(4 pt 1):E666-E675.

29. Postic C, et al. Dual roles for glucokinase in glucose homeostasis as determined by liver and pancreatic beta cell-specific gene knock-outs using Cre recombinase. J Biol Chem. 1999;274(1):305-315.

30. Walker AK, et al. Conserved role of SIRT1 orthologs in fasting-dependent inhibition of the lipid/ cholesterol regulator SREBP. Genes Dev. 2010; 
24(13):1403-1417

31. Giandomenico V, Simonsson M, Gronroos E, Ericsson J. Coactivator-dependent acetylation stabilizes members of the SREBP family of transcription factors. Mol Cell Biol. 2003;23(7):2587-2599.

32. Chiang JY. Bile acids: regulation of synthesis. J Lipid Res. 2009:50(10):1955-1966.

33. Cornu M, Modi H, Kawamori D, Kulkarni RN Joffraud M, Thorens B. Glucagon-like peptideincreases $\beta$-cell glucose competence and proliferation by translational induction of insulin-like growth factor-1 receptor expression. J Biol Chem. 2010;285(14):10538-10545.

34. Dufer $M$, et al. Bile acids acutely stimulate insulin secretion of mouse $\beta$-cells via farnesoid $X$ receptor activation and K(ATP) channel inhibition. Diabetes. 2012;61(6):1479-1489

35. Prawitt J, Caron S, Staels B. Bile acid metabolism and the pathogenesis of type 2 diabetes. Curr Diab Rep. 2011;11(3):160-166.

36. Shang Q, Liu MK, Saumoy M, Holst JJ, Salen $\mathrm{G}, \mathrm{Xu}$ G. The combination of colesevelam with sitagliptin enhances glycemic control in diabetic ZDF rat model. Am J Physiol Gastrointest Liver Physiol. 2012;302(8):G815-G823

37. Herrema $\mathrm{H}$, et al. Bile salt sequestration induces hepatic de novo lipogenesis through farnesoid $\mathrm{X}$ receptor- and liver $\mathrm{X}$ receptor alpha-controlled metabolic pathways in mice. Hepatology. 2010; 51(3):806-816

38. Shang Q, Saumoy M, Holst JJ, Salen G, Xu G. Colesevelam improves insulin resistance in a diet-induced obesity (F-DIO) rat model by increasing the release of GLP-1. Am J Physiol Gastrointest Liver Physiol. 2010;298(3):G419-G424

39. Russell DW. The enzymes, regulation, and genetics of bile acid synthesis. Annu Rev Biochem. 2003 72:137-174

40. Guillam M-T, et al. Early diabetes and abnormal postnatal pancreatic islet development in mice lacking GLUT2. Nat Genet. 1997;17(3):327-330

41. Selfridge J, Pow AM, McWhir J, Magin TM, Melton
DW. Gene targeting using a mouse HPRT minigene/HPRT-deficient embryonic stem cell system: inactivation of the ERCC-1 gene. Somat Cell Mol Genet. 1992;18(4):325-336.

42. Sinal CJ, Tohkin M, Miyata M, Ward JM, Lambert $\mathrm{G}$, Gonzalez FJ. Targeted disruption of the nuclear receptor FXR/BAR impairs bile acid and lipid homeostasis. Cell. 2000;102(6):731-744.

43. Selivanov SS, Picard Y, Cadorette J, Rodrigue S, Lecomte R. Detector response models for statistical iterative image reconstruction in high resolution PET. IEEE Trans Nucl Sci. 2000;47(22):1168-1175.

44. Thorens B, Cheng ZQ, Brown D, Lodish HF. Liver glucose transporter: a basolateral protein in hepatocytes and intestine and kidney cells. $A m J$ Physiol. 1990;259(6 pt 1):C279-C285.

45. Tian JM, Schibler U. Tissue-specific expression of the gene encoding hepatocyte nuclear factor 1 may involve hepatocyte nuclear factor 4. Genes Dev. 1991 5(12A):2225-2234

46. Folch J, Lees M, Sloane Stanley GH. A simple method for the isolation and purification of total lipides from animal tissues. J Biol Chem. 1957; 226(1):497-509.

47. Choi GT, Casu M, Gibbons WA. N.m.r. lipid profiles of cells, tissues and body fluids. Neutral, non -acidic and acidic phospholipid analysis of Bond Elut chromatographic fractions. Biochem J. 1993; 290(pt 3):717-721.

48. Minehira K, et al. Blocking VLDL secretion causes hepatic steatosis but does not affect peripheral lipid stores or insulin sensitivity in mice. J Lipid Res. 2008 ; 49(9):2038-2044

49. Chen JL, et al. Physiologic and pharmacologic factors influencing glyceroneogenic contribution to triacylglyceride glycerol measured by mass isotopomer distribution analysis. J Biol Chem. 2005; 280(27):25396-25402

50. Turner SM, et al. Measurement of TG synthesis and turnover in vivo by $2 \mathrm{H} 2 \mathrm{O}$ incorporation into the glycerol moiety and application of MIDA. Am J Physiol Endocrinol Metab. 2003;285(4):E790-E803.
51. Burcelin R, Eddouks M, Maury J, Kande J, Assan R, Girard J. Excessive glucose production, rather than insulin resistance, accounts for hyperglycemia in recent-onset streptozocin-diabetic rats. Diabetologia. 1995;38(3):283-290.

52. Tsao T-S, Burcelin R, Katz EB, Huang L, Charron MJ. Enhanced insulin action due to targeted GLUT4 overexpression exclusively in muscle. Diabetes. 1996;45(1):28-36.

53. Shen HQ, Zhu JS, Baron AD. Dose-response relationship of insulin to glucose fluxes in the awake and unrestrained mouse. Metabolism. 1999; 48(8):965-970

54. Kamohara R, Burcelin R, Halaas JL, Friedman JM, Charron MJ. Acute stimulation of glucose metabolism in mice by leptin treatment. Nature. 1997; 389(6649):374-377.

55. Preitner F, Mody N, Graham TE, Peroni OD, Kahn BB. Long-term Fenretinide treatment prevents high-fat diet-induced obesity, insulin resistance, and hepatic steatosis. Am J Physiol Endocrinol Metab. 2009;297(6):E1420-E1429.

56. Maechler P, Gjinovci A, Wollheim CB. Implication of glutamate in the kinetics of insulin secretion in rat and mouse perfused pancreas. Diabetes. 2002;51(suppl 1):S99-S102.

57. Preitner F, et al. Gluco-incretins control insulin secretion at multiple levels as revealed in mice lacking GLP-1 and GIP receptors. J Clin Invest. 2004; 113(4):635-645

58. R Development Core Team. R: A Language And Environment For Statistical Computing. Vienna, Austria: R Foundation for Statistical Computing; 2009.

59. Irizarry R, et al. Exploration, normalization, and summaries of high density oligonucleotide array probe level data. Biostatistics. 2003;4(2):249-264.

60. Smyth G. Linear models for microarray data. In: Gentleman R, Carey VJ, Huber W, Irizarry RA, Dudoit S, eds. Bioinformatics and Computational Biology Solutions using $R$ and Bioconductor (Statistics for Biology and Health). New York, New York, USA: Springer; 2005:397-420. 